\title{
Deformation and rigidity for group actions and von Neumann algebras
}

\author{
Sorin Popa
}

\begin{abstract}
We present some recent rigidity results for von Neumann algebras ( $\mathrm{II}_{1}$ factors) and equivalence relations arising from measure preserving actions of groups on probability spaces which satisfy a combination of deformation and rigidity properties. This includes strong rigidity results for factors with calculation of their fundamental group and cocycle superrigidity for actions with applications to orbit equivalence ergodic theory.
\end{abstract}

Mathematics Subject Classification (2000). Primary 46L35; Secondary 37A20, 22D25, 28 D15.

Keywords. von Neumann algebras, $\mathrm{II}_{1}$ factors, amenability and property $\mathrm{T}$ for groups, measure preserving actions, Bernoulli actions, orbit equivalence, cocycles.

\section{Introduction}

A measure preserving action $\Gamma \curvearrowright X$ of a countable group $\Gamma$ on a probability space $(X, \mu)$ gives rise in a natural way to a von Neumann algebra $L^{\infty}(X) \rtimes \Gamma$, through the group measure space (or crossed product) construction of Murray and von Neumann ([MvN36]). If $\Gamma$ is infinite and the action is free and ergodic then $L^{\infty}(X) \rtimes \Gamma$ is a $\mathrm{II}_{1}$ factor, a highly non-commutative infinite dimensional algebra with a positive trace. A central problem in the theory of von Neumann algebras is the classification up to isomorphisms of these factors in terms of their group/action data. Related to this, it was already shown in ([Si55]) that the isomorphism class of $L^{\infty}(X) \rtimes \Gamma$ only depends on the equivalence relation given by the orbits of $\Gamma \curvearrowright X$. This led to the study of actions of groups up to orbit equivalence ([D59]), an area in ergodic theory which since then developed in parallel but closely related to von Neumann algebras.

The early years concentrated on the amenable case, culminating with Connes' celebrated theorem that all $\mathrm{II}_{1}$ factors arising from actions of amenable groups are isomorphic ([C76]). Also, all ergodic actions of amenable groups on the non-atomic probability space were shown orbit equivalent in ([OW80], [CFW81]). But nonamenable groups were used to produce large families of non-isomorphic factors in ([MvN43], [D63], [Sch63], [Mc70], [C75]), indicating the richness of the theory. Rigidity phenomena started to unveil in the work of Connes ([C80]), who discovered that factors arising from groups with property $(\mathrm{T})$ of Kazhdan have countable outer

Proceedings of the International Congress of Mathematicians, Madrid, Spain, 2006

(C) 2007 European Mathematical Society 
automorphism and fundamental groups ([C80]). On the OE side, Zimmer obtained a cocycle superrigidity result for actions of higher rank semisimple Lie groups, a dynamical generalization of Margulis superrigidity which enabled him to prove that free ergodic actions of lattices such as $\mathrm{SL}(n, \mathbb{Z})$ are non-OE for different $n$ 's ([Z80]). Surprising non-embeddability results for $\mathrm{II}_{1}$ factors arising from certain lattices were then shown in ([CJ85], [CoH89]). More applications of all these ideas were derived in ([P86], [GoNe87], [GeGo88], [CoZ89]).

We present in this paper some recent progress made in these areas, triggered by the discovery in ([P01a], [P01b]) that if a group action $\Gamma \curvearrowright X$ satisfies both a rigidity condition (e.g. a weak form of property $\mathrm{T}$ ) and a deformability property (e.g. Haagerup property on the group, or Bernoulli-like malleability on the action), then the overall rigidity of $L^{\infty}(X) \rtimes \Gamma$ is considerably enhanced. Two new techniques, $d e$ formation/rigidity and intertwining subalgebras, were developed to exploit this idea ([P01b], [P03], [P04a]). This led to the first strong rigidity results in von Neumann algebra theory, showing that any isomorphism between factors arising from Bernoulli actions of Kazhdan groups comes from conjugacy of actions and isomorphism of groups ([P03], [P04a]). When combined with work of Gaboriau in OE ergodic theory ([G00], [G01]), it also led to the solution in ([P01b], [P03]) of long standing open problems of Murray-von Neumann ([MvN43]) and Kadison ([Ka67]) on the fundamental group of factors.

The von Neumann algebra framework and deformation/rigidity techniques also allowed proving cocycle superrigidity with arbitrary discrete groups as targets for all Bernoulli actions $\Gamma \curvearrowright\left(X_{0}, \mu_{0}\right)^{\Gamma}$, first in the case $\Gamma$ is Kazhdan ([P05]), then for $\Gamma$ a product between a non-amenable and an infinite group ([P06]). When applied to cocycles coming from $\mathrm{OE}$, this provided new $O E$ superrigidity results, showing that if in addition $\Gamma$ has no finite normal subgroups then any OE between a Bernoulli $\Gamma$-action and a free action $\Lambda \curvearrowright Y$ of an arbitrary countable group comes from a conjugacy. This added to the recent rigidity results obtained in OE ergodic theory using measure theoretic framework in ([Fu99a], [Fu99b], [G00], [G02], [MoS02]; see [S05] for a survey).

The presentation is organized as follows: In Sections 1, 2 we recall the basic definitions related to $\mathrm{II}_{1}$ factor framework and its specific analysis tools (c.p. maps and Hilbert bimodules, Jones basic construction). In Section 3 we discuss the rigidity statements we aim to prove and review past progress. Section 4 explains the use of "separability arguments" in rigidity results while Sections 5, 6 describe the intertwining and resp. deformation/rigidity techniques. In Sections 7-10 we state the results obtained through these techniques in ([P01b], [P03], [P04a], [IPeP05], [P05]).

\section{The $\mathrm{II}_{1}$ factor framework}

A von Neumann algebra is an algebra of linear bounded operators on a Hilbert space $\mathscr{H}$, containing $\operatorname{id}_{\mathscr{H}}$, closed under the adjoint $*$-operation and closed in the 
weak operator topology given by the seminorms $|\langle T \xi, \eta\rangle|, T \in \mathscr{B}(\mathcal{H}), \xi, \eta \in \mathcal{H}$. These conditions ensure that once an operator $T$ lies in the algebra so does its polar decomposition, and if in addition $T=T^{*}$ then the functional calculus of $T$ with Borel functions belongs to it as well. The algebra $\mathscr{B}(\mathscr{H})$ of all linear bounded operators on $\mathscr{H}$ is an example of a von Neumann algebra. If $\mathcal{X} \subset \mathscr{B}(\mathscr{H})$ is a selfadjoint subset (for instance the range of a unitary representation of a group) then the set $\mathcal{X}^{\prime}$ of operators $T \in \mathscr{B}(\mathscr{H})$ commuting with all elements in $\mathcal{X}$ is a von Neumann algebra. By a well known theorem of von Neumann, a $*$-algebra $M \subset \mathscr{B}(\mathcal{H})$ is a von Neumann algebra iff it is equal to its bicommutant $M^{\prime \prime}=\left(M^{\prime}\right)^{\prime}$.

1.1. von Neumann algebras from group actions. If $(X, \mu)$ is a standard probability space then the algebra of left multiplication operators by elements in $L^{\infty}(X)$ on the Hilbert space $L^{2}(X)$ is a von Neumann algebra. By the spectral theorem, any singly generated abelian von Neumann algebra $A$ is in fact of this form. One identifies (functorially) a measure preserving isomorphism of probability spaces $\Delta:(X, \mu) \simeq(Y, \nu)$ (defined a.e.) with the integral preserving algebra isomorphism $\Delta:\left(L^{\infty} X, \int \cdot \mathrm{d} \mu\right) \simeq$ $\left(L^{\infty} Y, \int \cdot \mathrm{d} \nu\right)$, via the relation $\Delta(x)(t)=x\left(\Delta^{-1}(t)\right)$ for all $x \in L^{\infty} X, t \in X$. In particular, this gives a canonical identification of the groups $\operatorname{Aut}(X, \mu), \operatorname{Aut}\left(L^{\infty}(X)\right.$, $\left.\int \cdot \mathrm{d} \mu\right)$.

Let now $\Gamma$ be a countable group and $\Gamma \curvearrowright X$ a measure preserving (m.p.) action of $\Gamma$ on the probability space $(X, \mu)$, viewed also as an action of $\Gamma$ on the algebra $L^{\infty}(X)$ preserving the integral, via the above identification. Consider the Hilbert space $\mathscr{H}=L^{2}(X) \bar{\otimes} \ell^{2} \Gamma$ and let $L^{\infty}(X)$ acting on it by left multiplication on the $L^{2}(X)$-component. Let also $\Gamma$ act on $\mathscr{H}$ as the multiple of the left regular representation $\lambda$, given by the unitary operators $u_{g}=\sigma_{g} \otimes \lambda_{g}, g \in \Gamma$, where $\sigma$ denotes the representation of $\Gamma$ on $L^{2}(X)$ extending $\Gamma \curvearrowright L^{\infty}(X)$. Let $M_{0}$ be the algebra generated by $L^{\infty}(X)$ and $\left\{u_{g}\right\}_{g}$ in $\mathcal{B}(\mathscr{H})$. The group measure space von Neumann algebra associated to $\Gamma \curvearrowright X$ is the weak closure of the algebra $M_{0}$ and is denoted $L^{\infty}(X) \rtimes \Gamma$.

It is convenient to view the dense subalgebra $M_{0} \subset M=L^{\infty} X \rtimes \Gamma$ as the algebra of "polynomials" $\Sigma_{g} a_{g} u_{g}$ with "coefficients" $a_{g}$ in $L^{\infty}(X)$, "indeterminates" (called canonical unitaries) $u_{g}, g \in \Gamma$, and multiplication rule $\left(a_{g} u_{g}\right)\left(a_{h} u_{h}\right)=$ $a_{g} \sigma_{g}\left(a_{h}\right) u_{g h}$, and to view $\mathscr{H}$ as the Hilbert space $\oplus_{g} L^{2}(X) u_{g}$ of square summable formal "Fourier series" $\Sigma_{g} \xi_{g} u_{g}$ with coefficients $\xi_{g}$ in $L^{2}(X)$. The same product formula gives an action of $M_{0}$ on $\mathscr{H}$ by left multiplication. In fact, if a series $x=\Sigma_{g} a_{g} u_{g} \in \mathscr{H}$ is so that its formal product with any element in $\Sigma_{h} \xi_{h} u_{h} \in \mathscr{H}$ remains in $\mathscr{H}$, then the left multiplication operator by $x$ lies in $M=\bar{M}_{0}^{w o}$, and any element of $M$ is of this form.

The particular case when $X$ is reduced to a point (i.e. $L^{\infty}(X)=\mathbb{C}$ ) of this construction gives the group von Neumann algebra $L \Gamma$ associated with $\Gamma$ ([MvN43]). It is naturally isomorphic to the von Neumann subalgebra of $L^{\infty}(X) \rtimes \Gamma$ generated by the canonical unitaries $\left\{u_{g}\right\}_{g}$. 
If we view $L^{\infty}(X)$ as a subalgebra of $M$ via the identification $a=a u_{e}=a 1$, then the integral $\int \cdot \mathrm{d} \mu$ extends to a functional $\tau$ on $M$, by $\tau(x)=\int x \mathrm{~d} \mu=\langle x, 1\rangle_{\mathscr{H}}=$ $\int a_{e} \mathrm{~d} \mu$, where $x=\Sigma_{g} a_{g} u_{g} \in M$. The functional $\tau$ is positive (i.e. $\tau\left(x^{*} x\right) \geq 0$ for all $x \in M$ ), faithful (i.e. $\tau\left(x^{*} x\right)=0$ iff $x=0$ ) and satisfies $\tau(x y)=\tau(y x)$ for all $x, y \in M$, i.e. it is a trace on $M$. Moreover, like the integral on $L^{\infty}(X), \tau$ is countably additive on mutually orthogonal projections, i.e. it is normal on $M$.

Like $L^{\infty}(X)$, which we view both as a subspace of $L^{2}(X)$ and as operators of left multiplication on $L^{2}(X)$, we view $x=\Sigma_{g} a_{g} u_{g} \in M$ both as an element in $\mathscr{H}$ and as operator of left multiplication on $\mathscr{H}$. The trace $\tau$ then recovers the Hilbert norm on $M \subset \mathscr{H}$ by $\|x\|_{2}=\tau\left(x^{*} x\right)^{1 / 2}$ and $\mathscr{H}$ is the completion of $M$ in this norm.

1.2. von Neumann algebras from equivalence relations. An action $\Gamma \curvearrowright X$ is free (i.e. $\mu(\{t \in X \mid g t=t\})=0$ for all $g \neq e)$ iff $L^{\infty}(X)$ is maximal abelian in $M$. If this is the case, then one can give the following alternative description of the von Neumann algebra $M=L^{\infty}(X) \rtimes \Gamma$, which only depends on the equivalence relations $\mathcal{R}_{\Gamma} \stackrel{\text { def }}{=}\{(t, g t) \mid t \in X, g \in \Gamma\}$ (cf. [Si55]): Let $m$ be the unique measure on $\mathcal{R}=\mathcal{R}_{\Gamma}$ satisfying $m\left(\left\{(t, g t) \mid t \in X_{0}\right\}\right)=\mu\left(X_{0}\right)$ for all $X_{0} \subset X$ and $g \in \Gamma$. If one identifies $\mathcal{R}$ with $X \times \Gamma$ via $(t, g t) \mapsto(t, g)$, then $m$ corresponds to the product measure on $X \times \Gamma$, so $L^{2}(\mathcal{R}, m)$ identifies naturally with $L^{2}(X) \otimes \ell^{2} \Gamma=\Sigma_{g} L^{2}(X) u_{g}$. Under this identification, if $x, y \in L^{2}(\mathcal{R}, m)$ then their formal product as elements in $\Sigma_{g} L^{2} X u_{g}$ corresponds to "matrix multiplication" $x y\left(t, t^{\prime}\right)=\Sigma_{s \sim t} x(t, s) y\left(s, t^{\prime}\right)$ for all $\left(t, t^{\prime}\right) \in \mathcal{R}$. Then $M$ is equal to the set $L(\mathcal{R})$ of operators on $L^{2}(\mathcal{R})$ for which there exists $x \in L^{2}(\mathcal{R})$ with (matrix) product $x y$ lying in $L^{2}(\mathcal{R})$, for any $y \in L^{2}(\mathcal{R})$. Note that $L^{\infty}(X)$ then corresponds to "matrices" $x$ that are supported on the diagonal $\{(t, t) \mid t \in X\} \subset \mathcal{R}$.

The above construction of the von Neumann algebra $L(\mathcal{R})$ works in fact for any equivalence relation $\mathcal{R}$ on $(X, \mu)$ that can be generated by a countable group $\Gamma \subset \operatorname{Aut}(X, \mu)$ (cf. [FM77]). Under this construction, $L^{\infty}(X)$ embeds in $L(\mathcal{R})$ as the subalgebra of "diagonal matrices" on $\mathcal{R}$, and is always maximal abelian in $L(\mathcal{R})$. Thus, unless $\Gamma$ acts freely, the construction of $L\left(\mathcal{R}_{\Gamma}\right)$ and $L^{\infty}(X) \rtimes \Gamma$ are different. For instance if $X$ is a one point set then $L^{\infty}(X) \rtimes \Gamma=L \Gamma$ while $L\left(\mathcal{R}_{\Gamma}\right)=\mathbb{C}$. The algebra $L(\mathcal{R})$ has a canonical trace, given by $\tau(x)=\langle x, 1\rangle=\int x_{0} \mathrm{~d} \mu$, where $x_{0}$ is the restriction of $x \in L^{2}(\mathcal{R})$ to the diagonal. Thus, $\tau$ is positive, normal, faithful and it extends $\int \cdot \mathrm{d} \mu$. If $\Gamma \curvearrowright X$ is not free then one still has a set of canonical unitaries $\left\{u_{g} \mid g \in \Gamma\right\}$ in $L\left(\mathcal{R}_{\Gamma}\right)$ which satisfy $u_{g} a u_{g}^{*}=g(a)$ for all $a \in L^{\infty}(X)$, and which together with $L^{\infty}(X)$ generate $L\left(\mathcal{R}_{\Gamma}\right)$, but with $\tau\left(u_{g}\right)$ being the measure of the set $\{t \in X \mid g t=t\}$ (thus possibly non-zero for $g \neq e$ ).

The maximal abelian subalgebra $A=L^{\infty}(X)$ in $M=L(\mathcal{R})$ has the property that the normalizer of $A$ in $M, \mathcal{N}_{M}(A)=\left\{u \in U(M) \mid u A u^{*}=A\right\}$, generates $M$ as a von Neumann algebra, i.e. $\mathcal{N}_{M}(A)^{\prime \prime}=M$ ([Di54]). Maximal abelian $*$-subalgebras $A \subset M$ in arbitrary $\mathrm{II}_{1}$ factors which satisfy this property are called Cartan subalge$\operatorname{bras}$ ([Ve71], [FM77]). It is shown in ([FM77]) that if $A \subset M$ is a Cartan subalgebra 
inclusion, then there exist a m.p. action $\Gamma \curvearrowright A=L^{\infty}(X, \mu)$ and a $U(A)$-valued 2-cocycle $v$ for the action such that $(A \subset M)=\left(L^{\infty}(X) \subset L(\mathcal{R}, v)\right)$, where the von Neumann algebra $L(\mathcal{R}, v)$ is defined similarly with $L(\mathcal{R})$ but with the product of canonical unitaries being twisted by the cocycle.

1.3. Conjugacy, orbit equivalence and algebra isomorphism. A conjugacy of group actions $\Gamma \curvearrowright(X, \mu), \Lambda \curvearrowright(Y, v)$ is an isomorphism of probability spaces $\Delta:(X, \mu) \simeq(Y, v)$ and a group isomorphism $\delta: \Gamma \simeq \Lambda$ such that $\delta(g) \circ \Delta=\Delta \circ g$ for all $g \in \Gamma$. If the actions are faithful (i.e. any $g \neq e$ implements a non-trivial automorphism) then the condition $\Delta \Gamma \Delta^{-1}=\Lambda$ is sufficient to ensure conjugacy. A conjugacy $(\Delta, \delta)$ implements an algebra isomorphism $\theta=\theta^{\Delta, \delta}: L^{\infty}(X) \rtimes \Gamma \simeq$ $L^{\infty}(Y) \rtimes \Lambda$, by $\theta\left(\Sigma_{g} a_{g} u_{g}\right)=\Sigma_{g} \Delta\left(a_{\delta(g)}\right) v_{\delta(g)}$, where $v_{h}$ are the canonical unitaries in $L^{\infty}(Y) \rtimes \Lambda$. Thus, $\theta$ extends $\Delta: L^{\infty}(X) \simeq L^{\infty}(Y)$.

By the construction in 1.2, an isomorphism $\Delta:(X, \mu) \simeq(Y, v)$, viewed as algebra isomorphism $\Delta: L^{\infty}(X) \simeq L^{\infty}(Y)$, extends to an isomorphism $L\left(\mathcal{R}_{\Gamma}\right) \simeq L\left(\mathcal{R}_{\Lambda}\right)$ iff $\Delta$ takes $\mathcal{R}_{\Gamma}$ onto $\mathcal{R}_{\Lambda}$, i.e. if it is an orbit equivalence (OE) of $\Gamma \curvearrowright X, \Lambda \curvearrowright Y$ (cf. [Si55], [FM77]). In other words, an OE of actions $\Gamma \curvearrowright X, \Lambda \curvearrowright Y$ is the same as an isomorphism of the associated von Neumann algebras $L\left(\mathcal{R}_{\Gamma}\right) \simeq L\left(\mathcal{R}_{\Lambda}\right)$ (or $L^{\infty}(X) \rtimes \Gamma \simeq L^{\infty}(Y) \rtimes \Lambda$ when actions are free) that takes $L^{\infty}(X)$ onto $L^{\infty}(Y)$. A third point of view, adopted in ([D59]; cf. also [Si55]), is to consider the full group $[\Gamma]$ of an action $\Gamma \curvearrowright X$ as the set of automorphisms $\alpha$ of $(X, \mu)$ for which there exists a (countable) partition of $X$ into measurable sets $X_{g}^{\alpha}, g \in \Gamma$ such that $\alpha$ coincides with $g$ on $X_{g}^{\alpha}$. Equivalently, $\alpha \in[\Gamma]$ iff the graph of $\alpha$ is contained in $\mathcal{R}_{\Gamma}$. It is then immediate to see that $\Delta: X \simeq Y$ is an OE of $\Gamma \curvearrowright X, \Lambda \curvearrowright Y$ iff $\Delta[\Gamma] \Delta^{-1}=[\Lambda]$, i.e. $\Delta$ conjugates the full groups.

Triggered this way by Murray-von Neumann group measure space construction, these observations led to the study of actions up to OE ([D59]), initiating what today is called orbit equivalence ergodic theory. The fact that $\mathrm{OE}$ of actions can be defined in both measure theoretic and von Neumann algebra terms allows a powerful dual approach to this subject. When adopting the point of view of studying group actions, an isomorphism of algebras $L^{\infty}(X) \rtimes \Gamma \simeq L^{\infty}(Y) \rtimes \Lambda\left(\right.$ or $\left.L\left(\mathcal{R}_{\Gamma}\right) \simeq L\left(\mathcal{R}_{\Lambda}\right)\right)$ is called a von Neumann equivalence (vNE) of $\Gamma \curvearrowright X, \Lambda \curvearrowright Y$. Thus, "conjugacy $\Rightarrow$ $\mathrm{OE} \Rightarrow \mathrm{vNE}$ ", but the reverse implications fail in general (cf. [D59], [CJ82]).

1.4. Ergodic actions and $\mathrm{II}_{\mathbf{1}}$ factors. A von Neumann algebra $M$ having a faithful normal trace $\tau$ is called finite. Thus $L^{\infty}(X) \rtimes \Gamma, L\left(\mathcal{R}_{\Gamma}\right)$ and their von Neumann subalgebras are finite.

A von Neumann algebra $M$ is a factor if its center, $\mathcal{Z}(M)=\{z \in M \mid z x=$ $x z$ for all $x \in M\}$, is equal to $\mathbb{C} 1_{M}$. A finite factor $(M, \tau)$ which has atoms (i.e. non-zero $p \in \mathcal{P}(M)$ with $p M p=\mathbb{C} p)$ is of the form $M \simeq \mathrm{M}_{n \times n}(\mathbb{C})$, for some $n$. A finite factor $M$ has no atoms (i.e. it is diffuse) iff it is infinite dimensional. $M$ is then called a type $\mathrm{II}_{1}$ factor. Like the algebra $\mathrm{M}_{n \times n}(\mathbb{C})$, a $\mathrm{II}_{1}$ factor has a unique trace $\tau$ with $\tau(1)=1$, and projections in $M$ can be conjugated by a unitary element in $M$ 
iff they have same trace. In other words, $\tau$ is a "dimension function" on $\mathcal{P}(M)$, but while $\tau(\mathcal{P}(M))=\{k / n \mid 0 \leq k \leq n\}$ when $M=\mathrm{M}_{n \times n}(\mathbb{C})$, for $\mathrm{II}_{1}$ factors the range of $\tau$ on projections is all the interval $[0,1]$.

The trace of a non-factorial finite von Neumann algebra $M$ is not unique, but $M$ does have a unique conditional expectation $\mathrm{Ctr}$ onto its center, called the central trace on $M$, which satisfies $\operatorname{Ctr}(x y)=\operatorname{Ctr}(y x)$ for all $x, y \in M$, is normal faithful and has the property that projections are unitary conjugate iff they have the same Ctr.

The algebra $L\left(\mathcal{R}_{\Gamma}\right)$ associated to an action $\Gamma \curvearrowright X$ is a factor iff $\Gamma$ acts ergodically on $(X, \mu)$, i.e., if $Z \subset X$ measurable satisfies $g(Z)=Z$ for all $g \in \Gamma$, then $\mu(Z)=$ 0,1 . If this is the case, then $L\left(\mathcal{R}_{\Gamma}\right)$ is $\mathrm{II}_{1}$ iff $X$ (or $\left.L^{\infty}(X)\right)$ has no atoms. In particular, if $\Gamma \curvearrowright X$ is free, then $L^{\infty}(X) \rtimes \Gamma$ is a factor iff $\Gamma \curvearrowright X$ is ergodic. It is a II $_{1}$ factor iff action is ergodic and $X$ diffuse (or $|\Gamma|=\infty$ ).

Given any countable (infinite) group $\Gamma$, its action on the non-atomic probability space by Bernoulli shifts, $\Gamma \curvearrowright\left(X_{0}, \mu_{0}\right)^{\Gamma} \simeq(\mathbb{T}, \mu)$, is free and ergodic (even mixing). More generally, if $\Gamma$ acts on a countable set $I$ then the action $\Gamma \curvearrowright\left(X_{0}, \mu_{0}\right)^{I}$, given by $g\left(t_{i}\right)_{i}=\left(t_{g-1}\right)_{i}$, is free whenever $\{i \mid g i \neq i\}$ is infinite for all $g \neq e$ and it is ergodic iff $|\Gamma i|=\infty$ for all $i \in I$, in which case it is even weak mixing, i.e. there exists $g_{n} \in \Gamma$ such that $\lim _{n} \mu\left(g_{n}(Z) \cap Z\right)=\mu(Z)^{2}$ for all $Z \subset X$. Such actions are called generalized Bernoulli actions.

If an action $\Gamma \curvearrowright X$ is not free, then a sufficient condition for $L^{\infty}(X) \rtimes \Gamma$ to be a $\mathrm{II}_{1}$ factor is that the action be ergodic and $\Gamma$ be infinite conjugacy class (ICC), i.e. $\left|\left\{g h g^{-1} \mid g \in \Gamma\right\}\right|=\infty$ for all $h \neq e$. In particular, $L \Gamma$ is a $\mathrm{II}_{1}$ factor iff $\Gamma$ is ICC. Examples of ICC groups are the infinite symmetric group $S_{\infty}$, the free groups $\mathbb{F}_{n}, 2 \leq n \leq \infty$, the groups $\operatorname{PSL}(n, \mathbb{Z}), n \geq 2$.

In fact, given any finite von Neumann algebra $(P, \tau)$ and an action $\Gamma \curvearrowright(P, \tau)$ of a countable group $\Gamma$ on it, one can construct the crossed product von Neumann algebra $P \rtimes \Gamma$ acting on the Hilbert space $\ell^{2}(\Gamma) \bar{\otimes} L^{2}(P)$, exactly as in the case $(P, \tau)=\left(L^{\infty}(X), \int \cdot \mathrm{d} \mu\right)$. Freeness of the action means in this general context that if $g \in \Gamma$ and $v \in P$ satisfy $v x=g(x) v$ for all $x \in P$, then either $g=e$ or $v=0$, and it is equivalent to the condition $P^{\prime} \cap P \rtimes \Gamma=\mathcal{Z}(P)$. If the action is free ergodic then the crossed product algebra is a factor.

Other constructions of factors are the tensor product and the free product (possibly with amalgamation) of finite factors $\left(P_{i}, \tau_{i}\right), i=1,2, \ldots$, which have selfexplanatory definitions. A useful framework for analysis arguments is the ultraproduct construction of $\mathrm{II}_{1}$ factors $\Pi_{\omega} M_{n}$, associated to a sequence of finite factors $M_{n}$, with $\operatorname{dim} M_{n} \rightarrow \infty$, and a free ultrafilter $\omega$ on $\mathbb{N}$ (e.g. [Mc70], [C76]).

1.5. Approximately finite dimensional $\mathrm{II}_{1}$ factors. Murray-von Neumann showed that all group measure space $\mathrm{II}_{1}$ factors arising from actions of locally finite groups, and more generally all approximately finite dimensional (AFD) $\mathrm{II}_{1}$ factors, are mutually isomorphic ([MvN43]). The unique AFD $\mathrm{II}_{1}$ factor, also called the hyperfinite $\mathrm{II}_{1}$ factor and denoted $R$, can be realized as the group factor $L\left(S_{\infty}\right)$, or as the infinite tensor product $\bar{\otimes}_{n}\left(\mathrm{M}_{2 \times 2}(\mathbb{C}) \text {, tr }\right)_{n}$. Since the relative commutant $P_{0}^{\prime} \cap M$ of any 
finite dimensional subfactor $P_{0}$ of a $\mathrm{II}_{1}$ factor $M$ is also a $\mathrm{II}_{1}$ factor, one can construct a copy of $R=\bar{\otimes}_{n}\left(\mathrm{M}_{2 \times 2}, \operatorname{tr}\right)_{n}$ inside any $\mathrm{II}_{1}$ factor $M$. A remarkable theorem of Connes shows that all amenable $\mathrm{II}_{1}$ factors are AFD and thus isomorphic to $R$ ([C76]). In particular $\mathrm{II}_{1}$ factors of the form $L^{\infty}(X) \rtimes \Gamma$ with $\Gamma$ amenable, and all their subfactors, are isomorphic to $R$. Similarly, by ([OW80], [CFW81]), all free ergodic actions of infinite amenable groups are OE and any two Cartan subalgebras of $R$ are conjugate by an automorphism of $R$. The outer automorphism group of $R$ is huge, in fact any separable locally compact group $\Gamma$ acts faithfully of $R$ by outer automorphisms (e.g. if $\Gamma$ is countable discrete then the "non-commutative" Bernoulli action $\Gamma \curvearrowright R=\bar{\otimes}_{g \in \Gamma}\left(\mathrm{M}_{2 \times 2}(\mathbb{C}), \operatorname{tr}\right)_{g}$ is properly outer) and the group of inner automorphisms $\operatorname{Int}(R)=\operatorname{Ad}(u) \mid u \in U(R)\}$ is dense in $\operatorname{Aut}(R)$, when the latter is endowed with the natural Polish group structure given by pointwise convergence in $\|\cdot\|_{2}$.

1.6. Amplifications and the fundamental group. The "continuous dimension" phenomenon allows defining the algebra of " $t$ by $t$ matrices" over a $\mathrm{II}_{1}$ factor $M$, or amplification of $M$ by $t, M^{t}$, for any positive real numbers $t$ : First note that if $p \in \mathcal{P}(M)$ then the algebra $p M p$, with normalized trace $\tau(\cdot) / \tau(p)$, is a $\mathrm{II}_{1}$ factor. Similarly if $n \geq 1$ is an integer then $\mathrm{M}_{n \times n}(M) \simeq \mathrm{M}_{n \times n}(\mathbb{C}) \otimes M$ is a $\mathrm{II}_{1}$ factor, with the normalized trace $\tau\left(\left(x_{i j}\right)_{i, j}\right)=\Sigma_{i} \tau\left(x_{i i}\right) / n$. With this in mind, for $t>0$ define $M^{t}$ to be the (isomorphism class of the) algebra $p \mathrm{M}_{n \times n}(M) p$, where $n \geq t$ is an integer and $p \in \mathrm{M}_{n \times n}(M)$ is a projection of (normalized) trace $t / n$.

Rather than studying only isomorphisms between $\mathrm{II}_{1}$ factors associated with actions, one considers stable isomorphisms (stable vNE) $L^{\infty}(X) \rtimes \Gamma \simeq\left(L^{\infty}(Y) \rtimes \Lambda\right)^{t}$ and respectively stable orbit equivalence $\mathcal{R}_{\Gamma} \simeq \mathcal{R}_{\Lambda}^{t}$ of actions $\Gamma \curvearrowright X, \Lambda \curvearrowright Y$, where $\mathcal{R}_{\Lambda}^{t}$ is the equivalence relation on a subset $Y_{0}$ of measure $t / n$ of $Y \times\{1,2, \ldots, n\}$ obtained by restriction to $Y_{0}$ of the product between $\mathcal{R}_{\Lambda}$ and the transitive relation on the $n$-point set, for some $n \geq t$. It is easy to see that $\mathcal{R}_{\Lambda}^{t}$ is itself implementable by a countable $\Lambda^{\prime} \subset \operatorname{Aut}\left(Y_{0}, \mu\right)$. We write $L^{\infty}(Y)^{t}$ for $L^{\infty}\left(Y_{0}\right)$.

Since both vNE and OE isomorphisms can be "amplified", stable vNE and OE are equivalence relations and $\left(M^{s}\right)^{t}=M^{s t}$. The fundamental group of a $\mathrm{II}_{1}$ factor $M$ (resp. of an equivalence relation $\mathcal{R}_{\Gamma}$ implemented by an ergodic action $\Gamma \curvearrowright X$ ) is defined by $\mathcal{F}(M) \stackrel{\text { def }}{=}\left\{t>0 \mid M^{t} \simeq M\right\}\left(\right.$ resp. $\left.\mathcal{F}\left(\mathcal{R}_{\Gamma}\right) \stackrel{\text { def }}{=}\left\{t>0 \mid \mathcal{R}_{\Gamma}^{t} \simeq \mathcal{R}_{\Gamma}\right\}\right)$.

An amplification of an AFD factor is clearly AFD. Thus, $\mathcal{F}(R)=\mathbb{R}_{+}^{*}$. Similarly, if $\mathcal{R}$ denotes the (unique) amenable equivalence relation implemented by any free ergodic action of an infinite amenable group, then $\mathcal{F}(\mathcal{R})=\mathbb{R}_{+}^{*}$.

1.7. Representations of finite algebras (Hilbert modules). If $(M, \tau)$ is a finite von Neumann algebra then $L^{2}(M)$ denotes the completion of $M$ in the norm $\|\cdot\|_{2}$ given by the trace, then $M$ can be represented on $L^{2}(M)$ by left multiplication operators, as a von Neumann algebra. This is the standard representation of $M$.

If $(M, \tau)=\left(L^{\infty} X, \int \cdot \mathrm{d} \mu\right)$ then $L^{2}(M)$ coincides with $L^{2}(X)$, and each $\xi \in$ $L^{2}(M)=L^{2}(X)$ can be viewed as the closed (densely defined) operator of (left) 
multiplication by $\xi$, with the spectral resolution of $|\xi|$ and the partial isometry $u=$ $\xi|\xi|^{-1}$ all lying in $L^{\infty}(X)$. For arbitrary $(M, \tau), L^{2}(M)$ can be similarly identified with the (densely defined) closed linear operators $\xi$ on $L^{2}(M)$, containing $M$ in their domain, with $|\xi|=\left(\xi^{*} \xi\right)^{1 / 2}$ having spectral resolution $e_{t}, t \geq 0$, in $M$ and $u=\xi|\xi|^{-1}$ in $M$ as well. The fact that the vector 1 is in the domain of $\xi$ is equivalent to $\xi$ being "square integrable", i.e. $\int t^{2} \mathrm{~d} \tau\left(e_{s}\right)<\infty$. Similarly, the completion of $M$ in the norm $\|x\|_{1}=\tau(|x|)$, denoted $L^{1}(M)$, can be identified with the space of closed linear operators $\xi$ on $M$ with polar decomposition $\xi=u|\xi|$ satisfying $u \in M$, $|\xi|^{1 / 2} \in L^{2}(M)$. One has $\left(L^{1}(M)\right)^{*}=M$, i.e. $L^{1}(M)$ is the predual of $M$.

In fact, $M$ acts on $L^{2}(M)$ by right multiplication as well, giving this way the standard representation of $M^{\text {op }}$ (the "opposite" of the algebra $M$ ). The left-right multiplication algebras $M, M^{\text {op }}$ commute, one being the commutant of the other. If one extends the antilinear isometric map $J_{M}(x)=x^{*}$ from $M$ to $L^{2}(M)$ by density, then $J_{M}^{2}=$ id and for each $x \in M$, viewed as left multiplication operator on $L^{2}(M), J_{M} x J_{M}$ gives the operator of right multiplication by $x^{*}$. Thus, $M^{\text {op }}=$ $J_{M} M J_{M}=M^{\prime}$.

Any other (separable) representation $M \subset \mathcal{B}(\mathcal{H})$ of $M$ as a von Neumann algebra (or left Hilbert $M$-module $\mathscr{H}$ ) is of the form $\mathscr{H} \simeq \oplus_{n} L^{2}(M) p_{n}$, for some $\left\{p_{n}\right\}_{n} \subset \mathcal{P}(M)$, with the action of $M$ by left multiplication. If $M$ is a factor, the number $\operatorname{dim}_{M} \mathscr{H} \stackrel{\text { def }}{=} \Sigma_{n} \tau\left(p_{n}\right) \in[0, \infty]$ characterizes the isomorphism class of $\mathscr{H}$. The $M$-module $\mathscr{H}$ can then be alternatively described as the (left) $M$-module $e_{11} L^{2}\left(\mathrm{M}_{n \times n}(M)\right) p$, where $n \geq \operatorname{dim} \mathscr{H}, e_{11} \in \mathrm{M}_{n \times n}(\mathbb{C})$ is a one dimensional projection and $p \in \mathcal{P}\left(\mathrm{M}_{n \times n}(M)\right)$ has (normalized) trace equal to $\operatorname{dim} \mathscr{H} / n$. Thus, the commutant $M^{\prime}$ of $M$ in $\mathscr{B}(\mathscr{H})$ is a $\mathrm{II}_{1}$ factor iff $\operatorname{dim}_{M} \mathscr{H}<\infty$ and if this is the case then $M^{\prime} \simeq\left(M^{t}\right)^{\mathrm{op}}$, where $t=\operatorname{dim}_{M} \mathscr{H}$. For general finite von Neumann algebra $M$, $M^{\prime}$ is finite iff $\Sigma_{n} \operatorname{Ctr}\left(p_{n}\right)$ is a densely defined operator in $\mathcal{Z}(M)$.

\section{Some $\mathrm{II}_{1}$ factor tools}

Thus, a free ergodic m.p. action $\Gamma \curvearrowright X$ of an infinite group on a non-atomic probability space gives rise to a $\mathrm{II}_{1}$ factor $M=L^{\infty}(X) \rtimes \Gamma$ with trace extending the integral on $L^{\infty}(X)$ and a natural pre-Hilbert space structure. Elements $x \in M$ have Fourier-like expansion, $x=\Sigma_{g} a_{g} u_{g}$, with coefficients in $L^{\infty}(X)$ and "basis" $\left\{u_{g}\right\}_{g}$ made out of unitary elements that satisfy $u_{g} u_{h}=u_{g h}$ and implement the $\Gamma$-action on $L^{\infty}(X)$, by $u_{g} a u_{g}^{*}=g(a)$. Spectral analysis and distribution behavior of elements in $M$ already reveal the dynamical properties of the group action. But in order to get proper insight into the structure of a $\mathrm{II}_{1}$ factor, for instance to recapture from the isomorphism class of $M=L^{\infty}(X) \rtimes \Gamma$ the initial building data $\Gamma \curvearrowright X$ (or part of it), we need to complement such local von Neumann algebra analysis tools with more global ones, some of which we briefly explain in this section. 
2.1. Hilbert bimodules and c.p. maps. While the Hilbert modules of a $\mathrm{II}_{1}$ factor $M$ reflect so nicely the continuous dimension phenomenon, they do not provide any actual insight into specific properties of $M$, the way a "good" representation theory should do. It was Connes who discovered, in the early $80 \mathrm{~s}$, that the appropriate representation theory for a $\mathrm{II}_{1}$ factor $M$ is given by the Hilbert $M$-bimodules, i.e. Hilbert spaces $\mathscr{H}$ with commuting von Neumann algebra representations $M, M^{\text {op }} \subset \mathscr{B}(\mathcal{H})$ ([C82]). Moreover, the same way the GNS construction provides an equivalence between unitary representations of a group and its positive definite functions, Hilbert $M$ bimodules can be "encoded" into a completely positive (c.p.) map $\phi: M \rightarrow M$, i.e. a linear map with all amplifications $\phi^{n}=\phi \otimes$ id on $\mathrm{M}_{n \times n}(M) \simeq M \otimes \mathrm{M}_{n \times n}(\mathbb{C})$, $n \geq 1$, positive.

This proved to be a deep, important idea at the conceptual level. Thus, ConnesJones used this point of view in ([CJ85]) to define the property (T) for abstract $\mathrm{II}_{1}$ factors $M$, by requiring: there is a finite $F \subset M$ and $\varepsilon>0$ such that if $\mathscr{H}$ has a unit vector with $\|y \xi-\xi y\| \leq \varepsilon$ for all $y \in F$, then $\mathscr{H}$ contains a non-zero central vector $\xi_{0}$, i.e. $x \xi_{0}=\xi_{0} x$ for all $x \in M$. They proved that this is equivalent to the following condition for c.p. maps: for all $\varepsilon_{0}$ there is a finite $F \subset M$ and $\delta>0$ such that if a subunital subtracial c.p. map $\phi: M \rightarrow M$ satisfies $\|\phi(y)-y\|_{2} \leq \delta$ for all $y \in F$, then $\|\phi(x)-x\|_{2} \leq \varepsilon$ for all $x$ in the unit ball $(M)_{1}$ of $M$. Moreover, they pointed out that all the representation theory of a group $\Gamma$ is reflected into the "representation theory" of a $\mathrm{II}_{1}$ factor $L^{\infty}(X) \rtimes \Gamma$ of the group action $\Gamma \curvearrowright X$, since any positive definite function $\varphi$ on $\Gamma$ gives rise to a c.p. $\operatorname{map} \phi=\phi_{\varphi}$, by $\phi\left(\Sigma a_{g} u_{g}\right)=\Sigma_{g} \varphi\left(a_{g}\right) u_{g}$. This led to a notion of Haagerup property for abstract $\mathrm{II}_{1}$ factors and the proof that property ( $\mathrm{T}$ ) $\mathrm{II}_{1}$ factors cannot be embedded into factors having this property ([CJ85]). It also led to several generalizations of all these notions in ([P86], [P01b]), as also explained in Section 6, and more recently to the construction of new cohomology theories for $\mathrm{II}_{1}$ factors ([CSh04], [Pe04]).

Note that any automorphism of $M$, and more generally endomorphism $\theta: M \rightarrow M$ (not necessarily unital), is subunital subtracial c.p. map. Thus, c.p. maps can be viewed as "generalized symmetries" of $M$, or as a very general notion of morphisms of $M$ into itself. This latter point of view was also present in work of Effros-Lance ([EL77]) and Haagerup ([H79], [CaH85], [CoH89]), and is now central to the theory of operator spaces. Altogether, c.p. maps, or equivalently Hilbert bimodules (also called correspondences by Connes [C82]) provide a key "global tool" in the study of $\mathrm{II}_{1}$ factors.

2.2. Subalgebras and the basic construction. The study of subalgebras of a $\mathrm{II}_{1}$ factor $M$ is an important part of the theory of von Neumann algebras, one of the most interesting aspects of which is Jones far reaching theory of subfactors ([J83], [J90]). For us here, the consideration of subalgebras of $M$ is mostly in relation to recovering the initial construction of $M$. For instance, if $\theta: L^{\infty}(X) \rtimes \Gamma \simeq L^{\infty}(Y) \rtimes \Lambda$ is an isomorphism, then we need to relate the positions of $P=\theta\left(L^{\infty}(X)\right)$ (resp. $P=\theta(L \Gamma))$ and $N=L^{\infty}(Y)($ resp. $N=L \Lambda)$ inside $M=L^{\infty}(Y) \rtimes \Lambda$. 
If $(M, \tau)$ is a finite von Neumann algebra then any von Neumann subalgebra $N$ of $M$ is finite, with $\tau_{\mid N}$ its faithful normal trace. The closure of $N$ in the Hilbert space $L^{2}(M)$ is clearly isomorphic to $L^{2}(N)$ and a Radon-Nikodym type theorem shows that the orthogonal projection $e_{N}$ of $L^{2}(M)$ onto $L^{2}(N) \subset L^{2}(M)$ takes $M$ (as a subspace of $\left.L^{2}(M)\right)$ onto $N$. The restriction of $e_{N}$ to $M$, denoted $E_{N}$, gives a $\tau$-preserving projection of $M$ onto $N$. Moreover $E_{N}\left(M_{+}\right)=N_{+}$and $E_{N}$ is $N$ bilinear, i.e. $E_{N}$ is a conditional expectation of $M$ onto $N$. Moreover, $E_{N}$ is the unique expectation which preserves $\tau$.

When viewed as operators on the Hilbert space $L^{2}(M), x \in M$ and $e_{N}$ satisfy the relations: $(a) e_{N} x e_{N}=E_{N}(x) e_{N} ;(b) x \in N$ iff $\left[x, e_{N}\right]=0$. Let $\left\langle M, e_{N}\right\rangle$ denote the von Neumann algebra generated in $\mathcal{B}\left(L^{2}(M)\right)$ by $M$ and $e_{N}$. Since we have $\vee\left\{x\left(e_{N}\left(L^{2}(M)\right)\right) \mid x \in M\right\}=L^{2}(M)$, from $(a),(b)$ it follows that $\operatorname{span} M e_{N} M$ is a *-algebra with support equal to 1 in $\mathscr{B}\left(L^{2}(M)\right)$. Thus, $\left\langle M, e_{N}\right\rangle=\overline{\operatorname{sp}}^{\mathrm{w}}\left\{x e_{N} y \mid\right.$ $x, y \in M\}$. Also, $\left\langle M, e_{N}\right\rangle=\left(J_{M} N J_{M}\right)^{\prime}, e_{N}\left\langle M, e_{N}\right\rangle e_{N}=N e_{N}$, implying that there are projections $p_{n} \nearrow 1$ in $\left\langle M, e_{N}\right\rangle$ such that $p_{n}\left\langle M, e_{N}\right\rangle p_{n}$ is a finite von Neumann algebra. Thus, $\left\langle M, e_{N}\right\rangle$ is a semifinite von Neumann algebra and it is finite iff $\operatorname{dim}_{N} L^{2}(M)<\infty$ (in the sense explained in 1.6). This is the Jones basic construction for $P \subset M$ ([J83]). The algebra $\left\langle M, e_{N}\right\rangle$ is endowed with a densely defined trace $\operatorname{Tr}$ by $\operatorname{Tr}\left(\Sigma_{i} x_{i} e_{N} y_{i}\right)=\Sigma_{i} \tau\left(x_{i} y_{i}\right)$, for $x_{i}, y_{i}$ finite sets of elements in $M$. One denotes $L^{2}\left(\left\langle M, e_{N}\right\rangle, \operatorname{Tr}\right)$ the completion of $\operatorname{sp} M e_{N} M$ in the norm $\|x\|_{2, \operatorname{Tr}}=$ $\operatorname{Tr}\left(x^{*} x\right)^{1 / 2}, x \in \operatorname{sp} M e_{N} M$.

Any Hilbert subspace of $L^{2}(M)$ which is invariant under multiplication to the right by elements in $N$ is a right Hilbert $N$-module (i.e. a left $N^{\mathrm{op}}$ module). If $\mathscr{H} \subset L^{2}(M)$ is a Hilbert subspace and $f$ is the orthogonal projection onto $\mathscr{H}$ then $\mathscr{H} N=\mathscr{H}$ (i.e. $\mathscr{H}$ is a right $N$-module) iff $f$ lies in $\left\langle M, e_{N}\right\rangle$. The right Hilbert $N$-module $\mathscr{H} \subset L^{2}(M)$ is invariant to multiplication from the left by a von Neumann subalgebra $P \subset M$, i.e. $P \mathscr{H} \subset \mathcal{H}$, iff $f \in P^{\prime} \cap\left\langle M, e_{N}\right\rangle$. Although all subalgebras we consider have infinite Jones index, the idea of viewing $L^{2}(M)$, as well as subspaces $\mathscr{H} \subset L^{2}(M)$ with $P \mathscr{H} N=\mathscr{H}$, as $P-N$ Hilbert bimodules, and the use of the basic construction as framework, came from developments in the theory of subfactors (e.g. [PiP86], [P86], [P94], [P97]).

\section{Prototype vNE and OE rigidity statements}

While all $\mathrm{II}_{1}$ factors (resp. equivalence relations) arising from ergodic actions of amenable groups look alike, in the non-amenable case the situation is very complex and rigidity phenomena were already detected at early stages of the subject ([MvN43], [D63], [Mc70], [C75], etc). Our aim in the rest of the paper is to investigate more "extreme" such rigidity phenomena, where for certain "small classes" of group actions the reverse of the implications "conjugacy $\Rightarrow \mathrm{OE} \Rightarrow \mathrm{vNE}$ " hold true as well. Typically, we fix a class of source group actions $\Gamma \curvearrowright(X, \mu)$ and a class of target actions $\Lambda \curvearrowright(Y, v)$, each of them characterized by a set of suitable assumptions, then attempt 
to show that the isomorphism of their group measure space algebras (vNE) entails isomorphism of the groups, or orbit equivalence of the actions (OE) or, ideally, the following type of statement:

(3.1). Under the given conditions on the source $\Gamma \curvearrowright X$ and target $\Lambda \curvearrowright Y$, if $\theta: L^{\infty}(X) \rtimes \Gamma \simeq\left(L^{\infty}(Y) \rtimes \Lambda\right)^{t}$, then $t=1$ and there exist a unitary element $u \in U\left(L^{\infty}(Y) \rtimes \Lambda\right), \gamma \in \operatorname{Hom}(\Lambda, \mathbb{T})$ and isomorphisms $\Delta: X \simeq Y, \delta: \Gamma \simeq \Lambda$ implementing a conjugacy of the actions, such that $\theta=\operatorname{Ad}(u) \circ \theta^{\gamma} \circ \theta^{\Delta, \delta}$, where $\theta^{\gamma}$ is the automorphism of $L^{\infty}(Y) \rtimes \Lambda$ implemented by $\gamma$ and $\theta^{\Delta, \delta}: L^{\infty}(X) \rtimes \Gamma \simeq$ $L^{\infty}(Y) \rtimes \Lambda$ the algebra isomorphism implemented by the conjugacy.

When the context concerns free ergodic actions, we call (3.1) a $v N E$ strong rigidity statement. While we do indeed obtain such results, other results will only go as far as showing that there exists a unitary element in the target algebra $u \in\left(L^{\infty}(Y) \rtimes \Lambda\right)^{t}$ such that $\operatorname{Ad}(u) \circ \theta$ takes $L^{\infty}(X)$ onto $L^{\infty}(Y)^{t}$, a type of result we call $v N E / O E$ rigidity.

Note that this latter statement is stronger than just showing vNE $\Rightarrow \mathrm{OE}$ for the specific classes of group actions involved. Indeed, this implication would only require proving there exists an automorphism $\theta_{0}$ of the target factor such that $\theta_{0} \circ \theta$ takes $L^{\infty}(X)$ onto $L^{\infty}(Y)$. While the vNE/OE version of (3.1) requires the $\theta_{0}$ to be inner! If the groups $\Gamma, \Lambda$ in (3.1) are amenable, when by ([C76]) the resulting group measure space factors are all isomorphic to $R$, there always exists an automorphism $\theta_{0}$ taking $L^{\infty}(X)$ onto $L^{\infty}(Y)$, by ([CFW81]), but there are uncountably many ways of decomposing $R$ as $L^{\infty}\left(X_{i}\right) \rtimes \mathbb{Z}$, with $\mathbb{Z} \curvearrowright X_{i}$ isomorphic actions, without the Cartan subalgebras $L^{\infty}\left(X_{i}\right) \subset R$ being unitarily conjugated in $R$ (cf. [FM77]).

The vNE/OE rigidity results can be further complemented with $\mathrm{OE}$ rigidity results from ergodic theory (e.g. [G01], [Fu99a], [MoSh02]), to recuperate the isomorphism class of the group, or derive that $t=1$. Taking the same action $\Gamma \curvearrowright X$ as source and target gives information about $\operatorname{Out}(M)=\operatorname{Aut}(M) / \operatorname{Int}(M)$ and $\mathcal{F}(M)$ for $M=$ $L^{\infty}(X) \rtimes \Gamma$.

If from the hypothesis of (3.1) we can only derive $\Gamma \simeq \Lambda$, then one calls it a $v N E$ rigidity result. The group algebra case of such a rigidity statement, with the assumptions on both groups to have property (T), corresponds to Connes Rigidity Conjecture ([C82]):

\section{(3.2). If $\Gamma, \Lambda$ are ICC property $(\mathrm{T})$ groups and $L \Gamma \simeq L \Lambda$ then $\Gamma \simeq \Lambda$}

The case when $X$ is a single point set of (3.1) then becomes the following stronger form of Connes's conjecture (see [J00]):

(3.2'). If $\Gamma$ is a property $(\mathrm{T})$ group, $\Lambda$ an arbitrary ICC group and $\theta: L \Gamma \simeq(L \Lambda)^{t}$, then $t=1$ and there exist a unitary $u \in U(L \Lambda)$ ), an isomorphism $\delta: \Gamma \simeq \Lambda$ and $\gamma \in \operatorname{Hom}(\Lambda, \mathbb{T})$ such that $\theta=\operatorname{Ad}(u) \circ \theta^{\gamma} \circ \theta^{\delta}$, where $\theta^{\gamma}$ is the automorphism of $L \Lambda$ implemented by $\gamma$ and $\theta^{\delta}: L \Gamma \simeq L \Lambda$ the isomorphism implemented by $\delta$.

Note that although $\left(3.2^{\prime}\right)$ assumes property $(\mathrm{T})$ only on the source group $\Gamma$, giving the statement a "superrigidity" flavor, the property (T) for $\Lambda$ is automatic from the 
isomorphism $L \Gamma \simeq(L \Lambda)^{t}$, due to results in ([CJ85]). The case $\Gamma=\Lambda$ in $\left(3.2^{\prime}\right)$ amounts to showing that $\operatorname{Out}(L \Gamma) \simeq \operatorname{Hom}(\Gamma, \mathbb{T}) \rtimes \operatorname{Out}(\Gamma)$ and $\mathcal{F}(L \Gamma)=\{1\}$. Connes' breakthrough rigidity result ([C80]), leading to his conjecture (3.2), shows that both groups are countable, being a verification "up to countable classes" of the formulas. To this date, the only other insight into Connes rigidity conjecture are the results of Connes-Jones, showing that $L(\operatorname{SL}(n, \mathbb{Z})), n \geq 3$, cannot be embedded into $L(\mathrm{SL}(2, \mathbb{Z}))([\mathrm{CJ} 85])$, and of Cowling-Haagerup, showing that if $\Gamma_{n}$ are lattices in $\operatorname{Sp}(n, 1)$ and $L\left(\Gamma_{n}\right) \subset L\left(\Gamma_{m}\right)$ then $n \leq m$ ([CoH89]).

If the isomorphism $\theta$ in (3.1) comes from a stable $\mathrm{OE}$ of free ergodic actions (so $\theta\left(L^{\infty}(X)\right)=\left(L^{\infty}(Y)\right)^{t}$ by hypothesis), then (3.1) amounts to deriving conjugacy from OE, a statement labeled OE strong rigidity in ([Fu99b], [MoSh02]). The "ideal" such statement is when there are no restrictions at all on the "target" side, in which case it qualifies as $O E$ superrigidity result. If only the isomorphism of the groups is derived, it is called $O E$ rigidity. This type of result already appeared in early 80 s, in pioneering work of Zimmer ([Z80], [Z84]). Thus, using his celebrated cocycle superrigidity theorem, itself a generalization of Margulis Superrigidity, he proved that free ergodic m.p. actions of the groups $\operatorname{SL}(n, \mathbb{Z}), n=2,3, \ldots$, are orbit inequivalent for different $n$ 's. Other OE rigidity results followed ([M82], [GeGo88]). By late 90s OE superrigidity phenomena started to unveil in the work of Furman, who added new ideas to the approach in ([Z91]) to derive that, more than just being rigid, actions of higher rank lattices such as $\operatorname{SL}(n, \mathbb{Z}) \curvearrowright \mathbb{T}^{n}, n \geq 3$, are in fact $O E$ superrigid, i.e. any orbit equivalence between such an action and an arbitrary free m.p. action of a discrete group $\Lambda$ comes from a conjugacy ([Fu99a, Fu99b]). Another important development on the $\mathrm{OE}$ side came with the work of Gaboriau who introduced a series of $\mathrm{OE}$ numerical-invariants for equivalence relations, allowing him to show that free m.p. actions of the free groups $\mathbb{F}_{n}$ are $\mathrm{OE}$ inequivalent for different $n \geq 1$ ([G00, G01]). A new set of $\mathrm{OE}$ superrigidity results was then established by Monod-Shalom, for doubly ergodic actions of products of word-hyperbolic groups ([MoS02]; see [Mo06], [S05] for survey articles).

These OE rigidity results were obtained by using a multitude of techniques, but all in measure theoretic framework. By 2001, vNE/OE rigidity started to emerge as well ([P01b]). Combined with ([G00]), they led to the first vNE rigidity results, where isomorphism of groups could be deduced from isomorphism of group measure space algebras, and factors with trivial fundamental group could be exhibited ([P01b]; see Section 7). Shortly after, vNE strong rigidity results of the form (3.1) were proved by using exclusively $\mathrm{II}_{1}$ factor framework ([P03], [P04a]; see Section 8). They provided completely new OE superrigidity results as well, obtained this time with von Neumann algebra methods ([P04a], [P05]; see Section 9; also [V06] for a combined presentation of these vNE and OE results). 


\section{Proving rigidity "up to countable classes"}

Before discussing the "precise" rigidity results mentioned above, we explain a method for deriving vNE and OE rigidity statements "up to countable classes", which grew out of Connes' initial rigidity paper ([C80]). Thus, by using the separability of the Hilbert space $L^{2}(M)$ of a $\mathrm{II}_{1}$ factor $M$ and the observation that two copies of the left regular representation $\lambda_{1}, \lambda_{2}$ of $\Gamma$ into $M$ that are sufficiently close on a set of generators of $\Gamma$ can be intertwined in $M$, it was shown in ([P86]) that in fact all $\mathrm{II}_{1}$ factors of the form $M=L^{\infty}(X) \rtimes \Gamma$, with $\Gamma$ a property (T) group, have countable fundamental group. Moreover, if the generators of a property (T) $\mathrm{II}_{1}$ subfactor $N_{1}$ of a separable $\mathrm{II}_{1}$ factor $M$ are almost contained into another subfactor $N_{2} \subset M$, then a "corner" of $N_{1}$ can be unitarily conjugated into $N_{2}$. By separability of $M$ this showed that $M$ contains at most countably many property (T) subfactors up to stable isomorphism ([P86]).

These are typical examples of what we call separability arguments, leading to rigidity statements "up to countable classes". They perfectly illustrate the power of the $\mathrm{II}_{1}$ factor framework. Such arguments were revived a few years ago, leading to new applications ([P01b], [Hj02], [Oz02], [GP03]). Thus, Hjorth proved that an infinite property (T) group $\Gamma$ has uncountably many non-OE actions ([Hj02]). Since by ([CW80], [Sc81]) any non-amenable, non(T) group has at least two non-OE free ergodic m.p. actions, this shows that any non-amenable group has at least two non-OE actions (in fact even non-vNE). His proof starts by noticing that a property (T) group $\Gamma$ has uncountably many non-conjugate actions $\sigma_{i}$ (using Gaussian actions to produce the family). If there exist only countably many equivalence relations, then there must be uncountably many $\sigma_{i}$ with same $\mathrm{OE}$ class $\mathcal{R}_{\Gamma}$. The $\sigma_{i}$ 's give copies $\lambda_{i}$ of the left regular representations in the normalizer of $L^{\infty}(X)$ in $L\left(\mathcal{R}_{\Gamma}\right)$. As in ([P86]), by separability there exist $i \neq j$ such that $\lambda_{i}$ and $\lambda_{j}$ can be intertwined by some $b \neq 0$ in $M=L\left(\mathcal{R}_{\Gamma}\right)$. Additional work shows that $b$ can be "pushed" into the normalizer of $L^{\infty}(X)$, implying that $\sigma_{i}, \sigma_{j}$ are conjugate, contradiction.

Another illustration is Ozawa's proof ([OzO2]) that there exists no separable $\mathrm{II}_{1}$ factor $M$ containing isomorphic copies of any separable $\mathrm{II}_{1}$ factor (not-necessarily with same unit as $M$ ): By a theorem of Gromov there exists a property (T) group $\Gamma$ with uncountably many non-isomorphic simple quotients $\Gamma_{i}$. Assuming there exists a separable $\mathrm{II}_{1}$ factor $M$ with $L\left(\Gamma_{i}\right) \subset M$ for all $i$, by the same separability argument as above for $\lambda_{i}$ 's the left regular representation of $\Gamma_{i}$, but all viewed as representations

of the group $\Gamma$, one gets an intertwiner between $\lambda_{i}, \lambda_{j}$ for some $i \neq j$. This implies $\Gamma_{i} \simeq \Gamma_{j}$, contradiction.

In this same vein, let us note that by using a theorem of Shalom in ([Sh00]), showing that any property ( $\mathrm{T}$ ) group is a quotient of a finitely presented property (T) group (only countably many of which exist), separability arguments as above show that Connes rigidity conjecture (3.2) does hold true "up to countable classes", i.e. the functor $\Gamma \mapsto L \Gamma$ on ICC property (T) groups is (at most) countable to 1 . Indeed, because if $M=L \Gamma_{i}$ for uncountably many non-isomorphic groups $\Gamma_{i}$, then 
by ([Sh00]) we may assume they are all quotients of the same property $(\mathrm{T})$ group $\Gamma$, and the previous separability argument gives a contradiction. Similarly one can prove that the strong rigidity conjecture $\left(3.2^{\prime}\right)$ holds true "modulo countable classes".

On the OE side, the same argument shows that there are at most countably many mutually measure equivalent (ME) property (T) groups (recall from [Fu1] that $\Gamma$ is ME to $\Lambda$ if there exist free ergodic m.p. actions $\Gamma \curvearrowright X, \Lambda \curvearrowright Y$ which are stably $\mathrm{OE})$. In other words, the following holds true "modulo countable classes": If two property $(\mathrm{T})$ groups are measure equivalent then they are virtually isomorphic.

A separability argument was also used to prove that free groups have uncountably many OE inequivalent actions, in ([GP03]): One starts by showing that for all $\mathbb{F}_{n} \curvearrowright X$ free, with one generator acting ergodically, there exists an increasing "continuous" family of sub-equivalence relations $\mathcal{R}_{\mathbb{F}_{n}, t} \subset \mathcal{R}_{\mathbb{F}_{n}}, 0<t \leq 1$, each one implemented by a free ergodic m.p. action of $\mathbb{F}_{n}$. Taking the initial $\mathbb{F}_{n}$-action to be the restriction to $\mathbb{F}_{n} \subset \operatorname{SL}(2, \mathbb{Z})$ of the natural action $\operatorname{SL}(2, \mathbb{Z}) \curvearrowright \mathbb{T}^{2}=\hat{\mathbb{Z}}^{2}$ and using the rigidity of this action (see 5.3), it follows from ([P01b]) that $\mathcal{R}_{\mathbb{F}_{n}, t}$ are rigid for $t \geq c$, for some $c<1$. Then a separability argument is used to show that $\mathcal{R}_{\mathbb{F}_{n}, t}, t \in[c, 1]$ (in fact even the $\mathrm{II}_{1}$ factors $\left.L\left(\mathcal{R}_{\mathbb{F}_{n}, t}\right)\right)$ are mutually non-isomorphic modulo countable classes.

Thus, separability arguments can be used to prove rigidity results in many situations, often without too much work, but they give the answer only "up to countable classes". However, such arguments brought up the following simple fact, specific to $\mathrm{II}_{1}$ factor framework, which is useful in proving "precise" rigidity results as well: If a group $\Gamma$ has property $(\mathrm{T})$, then all its representations into a $\mathrm{II}_{1}$ factor are isolated. More precisely, if $M$ is a $\mathrm{II}_{1}$ factor, $(F, \varepsilon)$ gives the critical neighborhood of the trivial representation of $\Gamma$ and $\pi_{i}: \Gamma \rightarrow \mathcal{U}\left(p_{i} M p_{i}\right), i=1,2$, satisfy $\left\|\pi_{1}(h)-\pi_{2}(h)\right\|_{2}<\varepsilon\left\|p_{1} p_{2}\right\|_{2}$ for all $h \in F$, then there exists a non-zero partial isometry $v$ in $M$ such that $\pi_{1}(g) v=v \pi_{2}(g)$ for all $g \in \Gamma$.

\section{Techniques for intertwining subalgebras}

Let $\theta: L^{\infty}(X) \rtimes \Gamma \simeq L^{\infty}(Y) \rtimes \Lambda$ be an isomorphism of $\mathrm{II}_{1}$ factors associated with free ergodic m.p. actions $\Gamma \curvearrowright X, \Lambda \curvearrowright Y$, as in (3.1). Denote by $M$ the target factor $L^{\infty}(Y) \rtimes \Lambda$, with $\left\{v_{h}\right\}_{h}$ its canonical unitaries, while $u_{g}$ denotes the canonical unitaries in the source factor $L^{\infty}(X) \rtimes \Gamma$. For simplicity, we identify $L^{\infty}(X) \rtimes \Gamma$ with $M$ via $\theta$. Proving a statement like (3.1) means finding a unitary $u \in M$ that conjugates $P=L^{\infty}(X)$ onto $N=L^{\infty}(Y)$, and possibly $\left\{u_{g}\right\}_{g}$ into $\left\{\mathbb{T} v_{h}\right\}_{h}$ (simultaneously!).

This is difficult even if we somehow know that $P, N$ are uniformly close one to another, but careful averaging techniques can be used to derive the desired conclusion. However, not being allowed "countable error", as in Section 4, there is no reason an isomorphism $\theta$ would take one structure close to the other, even on finite sets. This fact constitutes a major obstacle in getting "precise" vNE rigidity results. Moreover, 
the Connes-Jones example ([CJ82]), where a group of the form $\Gamma=\Gamma_{0} \times \Gamma_{1}$, with $\Gamma_{0}$ property (T) and $\Gamma_{1}$ an infinite product of non-commutative groups, is shown to have two non-OE free ergodic actions that give the same von Neumann algebra, suggests that $\mathrm{vNE}$ rigidity may in fact not occur.

Such problems were overcome in recent years due to a combination of two new ideas and sets of techniques, that we call deformation/rigidity and intertwining subalgebras (cf. [P01b], [P03], [P04a]).

The intertwining technique establishes some efficient criteria for deciding whether two von Neumann subalgebras $P, N$ of a $\mathrm{II}_{1}$ factor $M$ can be conjugated one into the other by a unitary element in $M$, or more generally if one can find $b \in M$ such that $p P p b \subset b N$, for some $p \in \mathcal{P}(P)$ with $p b \neq 0$. If such an "intertwiner" $b$ exists, we write $P \prec_{M} N$. Of equal importance in this problematic are the techniques for evaluating relative commutants $P^{\prime} \cap M$ and normalizers $\mathcal{N}(P)=\left\{u \in \mathcal{U}(M) \mid u P u^{*}=P\right\}$ of subalgebras $P \subset M$, which we view as part of the theory of "intertwining subalgebras".

The first criteria for whether $P \prec_{M} N$ and for estimating $P^{\prime} \cap M, \mathcal{N}(P)^{\prime \prime}$ appeared in ([P83]; see also [P91]), but for particular classes of factors $M$ and subalgebras. The general criteria below, which give several equivalent characterizations of $P \prec_{M} N$, is due to insight gained during 1983-1997 in the study of subalgebras of finite Jones index in ([PiP86], [P86], [P91], [P94], [P97]):

Theorem 5.1 ([P01b], [P03]). Let $(M, \tau)$ be a finite von Neumann algebra and $P, N \subset M$ von Neumann subalgebras. The following are equivalent:

(i) $P \prec_{M} N$.

(ii) There exists a Hilbert $P-N$ bimodule $\mathscr{H} \subset L^{2}(M)$ such that $\operatorname{dim} \mathscr{H}_{N}<\infty$.

(iii) There exists a non-zero projection $f \in P^{\prime} \cap\left\langle M, e_{N}\right\rangle$ such that $\operatorname{Tr}(f)<\infty$.

(iv) There exist projections $p \in P, q \in N$ a unital isomorphism $\psi: p P p \rightarrow q N q$ (not necessarily onto) and a partial isometry $v \in M$ such that $v v^{*} \in p P p^{\prime} \cap$ $p M p, v^{*} v \in \psi(p P p)^{\prime} \cap q M q$ and $x v=v \psi(x)$ for all $x \in p P p$.

Moreover, non-(i) is equivalent to:

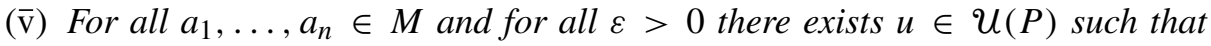
$\left\|E_{N}\left(a_{i} u a_{j}\right)\right\|_{2} \leq \varepsilon$ for all $i, j$.

This is the "core" result in the series of criteria which constitute the intertwining subalgebras techniques.

Condition $(\overline{\mathrm{v}})$ is very useful as a starting point in contradiction arguments. It is also useful in order to get some information about elements in the algebra $P$, in case one knows that $P$ cannot satisfy $P \prec_{M} N$, for instance because it cannot be embedded into $N$ (e.g. if say $N$ is abelian and $P$ is type $\mathrm{II}_{1}$ ). 
Condition (iv) is of course the one we seek when we expect $P$ to be unitary conjugated into $N$. It is however non-trivial to get from (iv) a unitary $u$ with $u P u^{*} \subset$ $N$, because one cannot apriori control the relative commutant of the image of the isomorphism $\psi$ (of which we only know it exists). This is solved on a case by case basis, by arguments in the spirit of ([P83]). It is in fact condition ( $\bar{v})$ that allows controlling the relative commutant of the algebra $P$, in many situations.

An important case when the relative commutants and normalizers can be accurately estimated is if $M=L^{\infty}(Y) \rtimes \Lambda$ comes from a free mixing action $\Lambda \curvearrowright Y$. Thus, if one denotes $N=L \Lambda$ and take a diffuse subalgebra $Q \subset N$, then $Q^{\prime} \cap M \subset N$, in fact all the normalizer of $Q$ in $M$ is contained in $N$. This implies that whenever $\psi, v$ are as in (iv) then $v^{*} v \in N$ and further work gives the unitary $u$ ([P03]). Other situations when this can be resolved is if $M=N \bar{\otimes} N_{0}=P \bar{\otimes} P_{0}$ ([OzP04]; see also 6.6 below), or if $M$ is a free product $M=N * N_{0}$ ([Oz04], [IPeP05]).

For general vNE rigidity problems it is particularly important to deal with the case when both $P, N$ are maximal abelian in $M$ (Cartan subalgebras). In this case one can overcome the "relative commutant issue" altogether, and get from (iv) a unitary element that conjugates $P$ onto $N$ ([P01b]). However, the proof of the (3.1)-type vNE strong rigidity result in ([P03], [P04a]; see Section 8) will only use the criterion for conjugating Cartan subalgebras after first showing that $L \Gamma, L \Lambda$ can be unitary conjugate onto each other, by using 5.1 and deformation/rigidity. Then $L \Gamma \prec_{M} L \Lambda$ is shown to imply $L^{\infty}(X)$ unitary conjugate to $L^{\infty}(Y)$, under no other assumptions but some good mixing conditions on the actions (see [P04a]). The proof of this implication does use a lot 5.1 and the criterion for conjugating Cartan subalgebras, but combined with lengthy, hard asymptotic analysis in the ultrapower factor $M^{\omega}$. It is the most difficult result in this series of intertwining techniques. One then proves that $L \Gamma \prec_{M} L \Lambda$ and $L^{\infty}(X) \prec_{M} L^{\infty}(Y)$ implies there exists $u \in U(M)$ that conjugates simultaneously $L^{\infty}(X)$ onto $L^{\infty}(Y)$ and $L \Gamma$ onto $L \Lambda$, which in turn implies $\operatorname{Ad} u$ takes $\left\{u_{g}\right\}_{g}$ into scalar multiples of $\left\{v_{h}\right\}_{h}$ as well.

Specific intertwining results can be obtained when the unit ball of $P$ is "almost contained" in a subalgebra $N$ of $M$, i.e. $\left\|E_{N}(x)-x\right\|_{2} \leq \varepsilon$ for all $x \in P,\|x\| \leq 1$, for some small $\varepsilon>0$. A pioneering such result appeared in ([Ch79]), where the basic construction framework $\left\langle M, e_{N}\right\rangle$ was for the first time used. Theorem 5.1 does cover also such cases, as $P$ almost contained in $N$ trivially implies $P^{\prime} \cap\left\langle M, e_{N}\right\rangle$ has finite non-zero projections. A detailed analysis of how to get from this a unitary conjugacy is carried out in ([P01b], [PSS04]), using subfactor methods.

\section{Deformation and rigidity in $\mathrm{II}_{1}$ factors}

As the discussion in the previous section shows, in order to recapture the building data of a group measure space factor, or at least part of it, it is sufficient to fit into one of the equivalent conditions of the criteria for intertwining subalgebras. For instance, to prove (3.1)-type results it is sufficient, by Section 5, to obtain finite dimensional 
$L^{\infty}(X)-L^{\infty}(Y)$ and $L \Gamma-L \Lambda$ Hilbert bimodules in $M=L^{\infty}(X) \rtimes \Gamma=L^{\infty}(Y) \rtimes \Lambda$.

Our strategy for producing such finite dimensional bimodules is to use deformability properties of the target group action and rigidity properties of the source group action. The method, which we call deformation/rigidity, works only when both conditions are met, taking a concrete technical form on a case by case basis. So first of all we need to single out classes of group actions whose associated factors satisfy both rigidity and deformability properties.

It is the $\mathrm{II}_{1}$ factor framework that makes this approach possible, as these algebras are particularly well adapted to deformations: by automorphisms, c.p. maps, completely bounded maps, etc. For us here, by a deformation of the identity $\operatorname{id}_{M}$ of a $\mathrm{II}_{1}$ factor $M$, we mean a sequence $\phi_{n}$ of subunital, subtracial, c.p. maps on $M$ such that $\lim _{n}\left\|\phi_{n}(x)-x\right\|_{2}=0$ for all $x \in M$. The maps $\phi_{n}$ of the deformations will frequently be automorphisms, but also conditional expectations and c.p. maps coming from positive definite functions on the group (for factors coming from group actions). The purpose of deformations is to reveal some "pole of rigidity" of the factor, i.e. a subalgebra $P$ that has a rigid position (in a sense or another) inside $M$. This roughly means that any deformation $\phi_{n}$ (often from a pre-assigned family of c.p. maps) must converge to $\operatorname{id}_{P}$ on the unit ball of $P$.

In order for the deformations to reveal the position of $P$ relative to $N$, besides $P \subset M$ being rigid we need $M$ to have "many" $N$-bilinear deformations, i.e. $M$ to be in some sense deformable ("soft") relative to $N$. In the end, we want that " $\phi_{n} \approx \mathrm{id}_{P}$ on $(P)_{1}$ " gives enough information so that, after some additional work, one gets from it a $P-N$ Hilbert bimodule $\mathscr{H} \subset L^{2}(M)$ with $\operatorname{dim} \mathscr{H}_{N}<\infty$. The "additional work" required may be minimal in some cases, like in examples 6.1, 6.6 and Section 7, or it may represent a substantial part of the argument, like in Example 6.2 and the results in Section 8-10. Moreover, the deformation/rigidity may require first an embedding of $M$ into a larger $\mathrm{II}_{1}$ factor $\tilde{M}$, then taking deformations of $\tilde{M}$ (e.g. by automorphisms, like in Sections 8-10). We illustrate this general strategy with a number of concrete situations where it has been be applied, postponing to the next sections the exact statement of the applications. The generic $\mathrm{II}_{1}$ factor $M$ involved in each situation is $M=L^{\infty}(X) \rtimes \Gamma=L^{\infty}(Y) \rtimes \Lambda$, as in (3.1), with the subalgebras $P, N \subset M$ being either $P=L^{\infty}(X), N=L^{\infty}(Y)$, or $P=L \Gamma, N=L \Lambda$.

6.1. Haagerup deformation and relative property $(\mathrm{T})([\mathrm{P01b}])$. $\mathrm{A} \mathrm{II}_{1}$ factor $M$ has property $\mathrm{H}$ (Haagerup property) relative to $N$ if there exists a deformation of $\operatorname{id}_{M}$ with subunital subtracial $N$-bilinear c.p. maps $\left\{\phi_{n}\right\}_{n}$ which are compact relative to $N$, i.e. for all $\left\{x_{k}\right\}_{k} \subset M$ with $E_{N}\left(x_{m}^{*} x_{m}\right) \leq 1$ and $\lim _{k}\left\|E_{N}\left(x_{k}^{*} x_{m}\right)\right\|_{2}=0$ for all $m$, one has $\lim _{m}\left\|\phi_{n}\left(x_{m}\right)\right\|_{2}=0$ (cf. [Cho83] for the case $N=\mathbb{C}$, [Bo93] in general). For subalgebras of the form $N \subset M=N \rtimes \Lambda$, this is equivalent to $\Lambda$ having the Haagerup approximation property ([H79]), i.e. there exist positive definite functions $\varphi_{n}$ on $\Lambda$ that tend pointwise to $1_{\Gamma}$ and vanish at $\infty\left(\varphi_{n} \in c_{0}(\Lambda)\right)$. The typical (nonamenable) example of groups with this property are the free groups $\mathbb{F}_{n}([\mathrm{H} 79])$, but 
all discrete subgroups in $\mathrm{SU}(n, 1), \mathrm{SO}(n, 1)$ have the property as well (see [CaH85]; also [CCJJV01] for a survey.)

An inclusion $P \subset M$ is rigid (or has the relative property (T)) if any deformation of $\operatorname{id}_{M}$ with subunital subtracial c.p. maps of $M$ tends uniformly to id $P$ on the unit ball of $(P)_{1}$ ([P01b]). For subalgebras of the form $P=L H \subset L G=M$, where $H \subset G$ are discrete groups, the rigidity of $L H \subset L G$ is equivalent to the relative property (T) of the inclusion $H \subset G$, as considered in ([K67], [Ma82]). (N.B. This property requires that unitary representations of $\Gamma$ which almost contain the trivial representation of $\Gamma$ must contain the trivial representation of $H$.) A well known example is the inclusion of groups $\mathbb{Z}^{2} \subset \mathbb{Z}^{2} \rtimes \mathrm{SL}(2, \mathbb{Z})$ ([K67], [Ma82]), more generally $\mathbb{Z}^{2} \subset \mathbb{Z}^{2} \rtimes \Gamma$ for any $\Gamma \subset \operatorname{SL}(2, \mathbb{Z})$ non-amenable $([\mathrm{Bu} 91])$.

With these concepts in hand, let us prove the result in ([P01b]), showing that if $P, N \subset M$ are so that $P \subset M$ is rigid and $M$ has property $\mathrm{H}$ relative to $N$ then $P \prec_{M} N$. Indeed, since $M$ has $N$-bilinear subunital subtracial c.p. maps $\phi$ that are compact relative to $N$ and close to $\operatorname{id}_{M}$, such $\phi$ follow uniformly close to $\operatorname{id}_{M}$ on $(P)_{1}$. If $P \nprec_{M} N$ then by 5.1 one can construct recursively $u_{k} \in U(P)$ such that $\lim _{k}\left\|E_{N}\left(u_{k}^{*} u_{m}\right)\right\|_{2}=0$ for all $m$. Thus, $\lim _{k}\left\|\phi_{n}\left(u_{k}\right)\right\|_{2}=0$ for all $n$ by compactness of $\phi_{n}$, while for large (but fixed) $k$ one has $\phi_{k}\left(u_{n}\right) \approx u_{n}$ uniformly in $n$, contradiction.

6.2. Malleability as source of intertwiners. Loosely speaking, a malleable deformation of a $\mathrm{II}_{1}$ factor $M$ over a subalgebra $N \subset M$ is an embedding of $M$ in a larger $\mathrm{II}_{1}$ factor $\tilde{M}$ and a continuous path $\alpha_{t}$ of automorphisms of $\tilde{M}$ such that $N$ is fixed by $\alpha_{t}$ for all $t$, with $\alpha_{0}=\mathrm{id}$ and $\alpha_{1}(M \ominus N) \perp(M \ominus N)$ as (pre)Hilbert spaces. More generally, one can merely require the automorphism $\alpha_{1}$ satisfying the above conditions to be in the connected component of $\operatorname{id}_{\tilde{M}} \operatorname{in}_{\operatorname{Aut}}(\tilde{M})=\left\{\rho \in \operatorname{Aut}(\tilde{M}) \mid N \subset \tilde{M}^{\rho}\right\}$. The interest of such deformations is that if $P \subset M$ is a subalgebra for which there exists $b \in \tilde{M}$ non-zero with $P b \subset b \alpha_{1}(P)$ (i.e. $\left.P \prec \tilde{M} \alpha_{1}(P)\right)$ and the intertwiner $b$ can be shown to belong to the $M$-bimodule $L^{2}\left(\operatorname{sp} M \alpha_{1}(M)\right.$, then $P \prec_{M} N$, more precisely a reinterpretation of $b$ as an element in the $M$-bimodule $L^{2}\left(\left\langle M, e_{N}\right\rangle, \operatorname{Tr}\right)$ produces a (non-zero) finite dimensional $P-N$ bimodule, which by 5.1 means $P \prec_{M} N$.

Thus, such deformations can be used to obtain unitary conjugacy between subalgebras of $M$, provided one can show that the continuous path $\alpha_{t}(P)$ in $\tilde{M}$ produces an intertwiner between $P$ and $\alpha_{1}(P)$. If the path is uniformly continuous on $P$ then by the remarks at the end of Sections 4 and 5 one can find intertwiners between $\alpha_{t}(P)$ and $\alpha_{t+\mathrm{d} t}(P)$, for "incremental" $\mathrm{d} t$, then try to patch them. To have uniform continuity and do the patching certain assumptions must be made, as explained below.

6.3. Malleability and property (T) ([P01a], [P03], [P04a]). Malleability properties and their importance in studying group actions and algebras were discovered in (2.1 of [P01a]), inspired by some considerations in (4.3.2 of [P86]). The formal way the concept is defined in (2.1 in [P01a] and 1.4, 1.5 in [P03]) is as follows: An 
action $\Lambda \curvearrowright(B, \tau)$ is malleable if there exist an embedding $B \subset \tilde{B}$ with an action $\Lambda \curvearrowright \tilde{B}$ extending $\Lambda \curvearrowright B$, and a continuous path $\alpha_{t}$ of automorphisms of $\tilde{B}$ commuting with the $\Lambda$-action, such that $\alpha_{0}=\mathrm{id}_{\tilde{B}}, \alpha_{1}(B) \perp B$ (in the sense of [P83]) and $\tilde{B}=\overline{\operatorname{sp}} B \alpha_{1}(B)$. In case $B=L^{\infty}(Y)$ this amounts to $\tilde{B} \simeq L^{\infty}(Y) \bar{\otimes} L^{\infty}(Y)$ with $\Lambda \curvearrowright L^{\infty}(Y) \otimes L^{\infty}(Y)$ the "double" action. Such deformations $\alpha_{t}$ implement automorphisms of $\tilde{M} \stackrel{\text { def }}{=} \tilde{B} \rtimes \Lambda$, still denoted $\alpha_{t}$, so this does fit into the general framework 6.2 for $M=B \rtimes \Lambda, N=L \Lambda$. Moreover, the condition $\operatorname{sp} B \alpha_{1}(B)$ dense in $\tilde{B}$ implies $\operatorname{sp} M \alpha_{1}(M)$ dense in $\tilde{M}$, insuring that any intertwiner of $P, \alpha_{1}(P)$ in $\tilde{M}$ produces an intertwiner of $P, N$ in $M$.

Examples of actions satisfying the malleability condition are all generalized Bernoulli actions $\Lambda \curvearrowright \mathbb{T}^{I}$, corresponding to some action of $\Lambda$ on a countable set $I$, and the non-commutative Bernoulli and Bogoliubov actions on the hyperfinite $\mathrm{II}_{1}$ factor $R$ ([P01a], [P03]). It was recently noticed in ([Fu06]) that Gaussian actions are malleable as well (see [CW81] or [CCJJV01] for the definition of such actions).

More "lax" conditions for malleable deformations, where $\operatorname{sp} B \alpha_{1}(B)$ is no longer required dense in $\tilde{B}$, were considered in $(4.2$ of [P03]) and (6.1 of [P01a]). In such cases, an element $b$ intertwining $P$ into $\alpha_{1}(P)$ can be shown to belong to $L^{2}\left(\operatorname{sp} M \alpha_{1}(M)\right)$ whenever some appropriate relative weak mixing condition of $\Lambda \curvearrowright$ $\tilde{B}$ wrt $\Lambda \curvearrowright L^{2}\left(\operatorname{sp} B \alpha_{1}(B)\right)$ holds true (e.g. 4.2.1 in [P03], 2.9 in [P05], 6.1 in [P01a]). All generalized Bernoulli actions $\Lambda \curvearrowright\left(B_{0}, \tau_{0}\right)^{I}$, with $B_{0}$ an arbitrary finite, AFD (equivalently amenable, by [C76]) von Neumann algebra, have malleable deformations satisfying the relative weak mixing requirement. Same for the "free Bernoulli actions" $\Lambda \curvearrowright\left(B_{0}, \tau_{0}\right)^{* I}=*_{i \in I}\left(B_{0}, \tau_{0}\right)_{i}$ with AFD base $\left(B_{0}, \tau_{0}\right)$ (cf. 6.1 in [P01a]).

Typically, if $\Lambda \curvearrowright B$ is a malleable action as above, or more generally $M$ is malleable over $N$, corresponding to $M=B \rtimes \Lambda, N=L \Lambda$, the rigidity condition required on the subalgebra $P \subset M$ is the relative property (T) as defined in 6.1. If $P=L \Gamma$, where $\Gamma \curvearrowright X$ is another action with $M=L^{\infty}(Y) \rtimes \Lambda=L^{\infty}(X) \rtimes \Gamma$ as in (3.1), one requires that $\Gamma$ itself has property (T). By rigidity, $\alpha_{t}(P)$ and $\alpha_{t+\mathrm{d} t}(P)$ are then uniformly close, for "incremental" $\mathrm{d} t$, so there is a non-zero intertwiner between them (for groups, this is trivial by the observations in Section 4). Patching these intertwiners gives an intertwiner between $P$ and $\alpha_{1}(P)$, which from the above shows that $P=L \Gamma$ can be intertwined into $N=L \Lambda$. But there are actually big difficulties in doing the "patching", as the intertwiners are apriori partial isometries and not unitaries, so when gluing them repeatedly we may end up getting 0 . The malleable deformations with a symmetry, called s-malleable, were introduced in (2.1, 6.1 of [P01a], 1.4 of [P03]) to overcome this issue. All above examples carry natural such symmetry, and so do the "free"-deformations found in ([IPeP05). In turn, much less than property (T) for $\Gamma$ is enough to obtain an intertwiner between $L \Gamma$ and $L \Lambda$ through this argument. Thus, if the $\Lambda$-action is mixing then it is sufficient that $\Gamma$ has an infinite subgroup $H \subset \Gamma$ with the relative property (T) and some weak normality condition, for instance existence of a chain of normal inclusions from $H$ to $\Gamma$ ( $H$ is w-normal in $\Gamma$ ). 
6.4. Malleability in free product algebras. A malleable deformation was also used in ([P01a]) to prove a cocycle rigidity result for actions of property (T) groups $\Gamma$ on $B=L \mathbb{F}_{\Gamma} \simeq L \mathbb{F}_{\infty}$, by free Bernoulli shifts. Inspired by (4.3.2 of [P86]) it gives an explicit construction of an action $\alpha$ of $\mathbb{R}$ on $\tilde{B}=B * B$ which commutes with the double action of $\Gamma$ on $\tilde{B}$ and checks $\alpha_{1}(B * \mathbb{C})=\mathbb{C} * B$. The cocycle rigidity is proved by using the general scheme 6.2 , with $M=B \rtimes \Gamma, \tilde{M}=\tilde{B} \rtimes \Gamma, N=L \Gamma$ and the natural extension to $\tilde{M}$ of the deformation $\alpha_{t}$. But this malleable deformation no longer satisfies the "tight" generating condition $\operatorname{sp} B \alpha_{1}(B)$ dense in $\tilde{B}$, and "descending" the intertwiner between $B, \alpha_{1}(B)$ from $\tilde{B}$ to $L^{2}\left(B \alpha_{1}(B)\right)$ requires more work.

Another malleable deformation in free product framework was discovered in ([IPeP05]), but for the acting group rather than for the action. Thus, let $\Gamma=\Gamma_{1} * \Gamma_{2} \curvearrowright$ $(B, \tau)$ and denote $M=B \rtimes \Gamma, \tilde{M}=B \rtimes\left(\Gamma * \mathbb{F}_{2}\right)$, with $\mathbb{F}_{2}$ acting trivially on $B$. Then $\tilde{M}$ can also be viewed as the amalgamated free product $\left(B \rtimes \Gamma_{1} * \mathbb{Z}\right) *_{B}\left(B \rtimes \Gamma_{1} * \mathbb{Z}\right)$. If $u_{1} \in L(\mathbb{Z} * 1) \subset L\left(\mathbb{F}_{2}\right), u_{2} \in L(1 * \mathbb{Z}) \subset L\left(\mathbb{F}_{2}\right)$ are the canonical generating unitaries and $h_{j}=h_{j}^{*}$ are so that $\exp \left(i h_{j}\right)=u_{j}$, then $h_{j}$ commute with $B$. Thus $\alpha_{t}=\operatorname{Ad}\left(\exp \left(i t h_{1}\right)\right) *_{B} \operatorname{Ad}\left(\exp \left(i t h_{2}\right)\right)$ implements an action of $\mathbb{R}$ on $\tilde{M}$ leaving $B$ fixed and satisfying $\alpha_{1}=\operatorname{Ad}\left(u_{1}\right) * \operatorname{Ad}\left(u_{2}\right), \alpha_{1}(L \Gamma)=u_{1} L \Gamma_{1} u_{1}^{*} * u_{2} L \Gamma_{2} u_{2}^{*} \perp L \Gamma$.

6.5. A general notion of rigidity for subalgebras. The above considerations justify considering the following:

Definition 6.5.1 ([P04b]). Let $M$ be a $\mathrm{II}_{1}$ factor, $P \subset M$ a von Neumann subalgebra and $\mathcal{L}$ a family of subunital subtracial c.p. maps of $M$. We say that the inclusion $Q \subset M$ is rigid with respect to (wrt) $\mathcal{L}$ if given any deformation of $\mathrm{id}_{M}$ by c.p. maps $\phi_{n} \in \mathcal{L}$ we have $\left\|\phi_{n}(x)-x\right\|_{2} \rightarrow 0$ uniformly for $x \in(P)_{1}$.

Note that the rigidity of $P \subset M$ as defined in 6.1 amounts to condition 6.5 .1 for $\mathcal{L}$ the family of all subunital subtracial c.p. maps on $M$, the particular case $P=M$ of which amounts to the Conne-Jones definition of property (T) for the $\mathrm{II}_{1}$ factor $M$ ([CJ85]). Also, the malleability/rigidity arguments above only used the rigidity of the inclusion $P \subset \tilde{M}$ wrt $\mathcal{L}=\left\{\alpha_{t}\right\}_{t} \subset \operatorname{Aut}(\tilde{M})$, where $\alpha$ is the malleable deformation.

Besides its rôle in deformation/rigidity approach to proving statements such as (3.1), the idea of defining various notions of rigidity for inclusions of algebras $P \subset M$ has other applications as well. Thus, if the family $\mathcal{L}$ has an abstract description, depending only on the isomorphism class of $P \subset M$, then the rigidity of $P \subset M$ wrt $\mathcal{L}$ is, of course, an isomorphism invariant for $P \subset M$. This point of view gives rise to useful applications to OE ergodic theory. For instance, a new OE invariant for a free ergodic m.p. action $\Gamma \curvearrowright X$ (more generally for equivalence relations $\mathcal{R}_{\Gamma}$ ) was defined in ([P01b]) by requiring that the inclusion $L^{\infty}(X) \subset L^{\infty}(X) \rtimes \Gamma\left(\operatorname{resp} L^{\infty}(X) \subset\right.$ $L\left(\mathcal{R}_{\Gamma}\right)$ ) is rigid (see also [PeP04]). From 6.1 it follows that if $\Gamma \subset \mathrm{SL}(2, \mathbb{Z})$ is nonamenable then $\Gamma \curvearrowright \mathbb{T}^{2}=\hat{\mathbb{Z}}^{2}$ has this property. More examples were constructed in ([Va05]). This notion of relative property (T) for free ergodic actions played an important rôle in the proof that free groups have uncountably many OE-inequivalent 
actions in ([GP03]). Another particular case of 6.5.1 recovers a concept considered in ([An87], [P86]): $\mathrm{A} \mathrm{II}_{1}$ factor $M$ has the property (T) relative to a subalgebra $P \subset M$ (or $P$ is co-rigid in $M$ ) if $M \subset M$ is rigid wrt the family of all subunital subtracial $P$-bilinear c.p. maps on $M$. Again, by the definition, this notion is an isomorphism invariant for the inclusion $P \subset M$, so in case $P=L^{\infty}(Y) \subset L^{\infty}(Y) \rtimes \Lambda=M$, it gives an OE invariant for the free action $\Lambda \curvearrowright Y$. For such inclusions, it is shown in ([P86]) that co-rigidity is equivalent to the property (T) of $\Lambda$.

6.6. Example ([P04c]). We end this discussion with a simple but very suggestive example related to definition 6.5.1: Let $M$ be a $\mathrm{II}_{1}$ factor of the form $M=Q \bar{\otimes} R$, where $R$ is the hyperfinite $\mathrm{II}_{1}$ factor and $Q$ is a non $(\Gamma) \mathrm{II}_{1}$ factor ([MvN43]). Let $\mathcal{L}$ be the family of all conditional expectations onto non $(\Gamma) \mathrm{II}_{1}$ subfactors $P \subset M$ with the property that $P^{\prime} \cap M \simeq R$ and $M=P \vee\left(P^{\prime} \cap M\right)$. It is easy to see that $Q \subset M$ is rigid wrt $\mathcal{L}$. Recovering the building data means in this case to show that if $M=P_{0} \otimes R_{0}$ is another decomposition with $P_{0} \operatorname{non}(\Gamma)$ and $R_{0} \simeq R$ then there exists a unitary element $u \in M$ such that $u P_{0} u^{*}=Q^{t}, u R_{0} u^{*}=R^{1 / t}$, for some $t>0$, where the identification $Q \bar{\otimes} R=Q^{t} \bar{\otimes} R^{1 / t}$ is self-explanatory. To prove this, one takes $P_{n}=P_{0} \otimes \mathrm{M}_{2^{n} \times 2^{n}}(\mathbb{C})$ where $\mathrm{M}_{2^{n} \times 2^{n}}(\mathbb{C}) \nearrow R_{0}$ and apply the rigidity of $Q \subset M$ with respect to the deformation $E_{P_{n}}$ to conclude that for a large enough $n$, the unit ball of $Q$ is almost contained in $P_{n}$. By the intertwining criteria 5.1, there exists $u \in U(M)$ that conjugates $Q$ into $P_{n}$, which from the split-off conditions implies the result.

Similarly, one can show that if $M$ is a $\mathrm{II}_{1}$ factor with $Q \subset M$ a von Neumann algebra such that $\{\operatorname{Ad}(u) \mid u \in U(Q)\}$ has spectral gap wrt $Q^{\prime} \cap M$ (i.e. there exist $u_{1}, \ldots, u_{n} \in \mathcal{U}(Q)$ and $c>0$ such that $\Sigma_{i}\left\|u_{i} x u_{i}^{*}-x\right\|_{2} \geq c\|x\|_{2}$ for all $x \in Q^{\prime} \cap M^{\perp}$ ), then $Q^{\prime} \cap M \subset M$ is rigid wrt $\mathscr{L}=\operatorname{Aut}(M)$ ([P06]).

\section{7. $\mathrm{vNE} / \mathrm{OE}$ rigidity from relative property $(\mathrm{T})$ and $\mathrm{H}$}

We formally state here the result discussed in 6.1, which uses Haagerup-type deformation of the acting group and relative property $(\mathrm{T})$ of the action. This is a typical $\mathrm{vNE} / \mathrm{OE}$ rigidity result, in the terminology established in (3.1), as it shows that for a certain class of group measure space $\mathrm{II}_{1}$ factors, any algebra isomorphism comes from an orbit equivalence of the actions, modulo perturbation by an inner automorphism.

Theorem 7.1 ([P01b]). Let $\Gamma \curvearrowright(X, \mu), \Lambda \curvearrowright(Y, v)$ be ergodic (not necessarily free) m.p. actions and assume $L^{\infty}(X) \subset L\left(\mathcal{R}_{\Gamma}\right)$ is rigid while $L\left(\mathcal{R}_{\Lambda}\right)$ has property $\mathrm{H}$ relative to $L^{\infty}(Y)$. If $\theta: L\left(\mathcal{R}_{\Gamma}\right) \simeq L\left(\mathcal{R}_{\Lambda}\right)$ is an algebra isomorphism then there exists $u \in \mathcal{U}\left(L\left(\mathcal{R}_{\Lambda}\right)\right)$ such that $\operatorname{Ad}(u)\left(\theta\left(L^{\infty}(X)\right)\right)=L^{\infty}(Y)$.

The next corollary lists some concrete examples when the assumptions of 7.1 are satisfied. 
Corollary 7.2. Let $\Gamma \subset \operatorname{SL}(2, \mathbb{Z})$ be a non-amenable subgroup and $\Gamma \curvearrowright \mathbb{T}^{2}=\hat{\mathbb{Z}}^{2}$ the action induced by the restriction to $\Gamma$ of the $\operatorname{SL}(2, \mathbb{Z})$ action on $\mathbb{Z}^{2}$. Let $\Lambda \curvearrowright(Y, v)$ be a free ergodic m.p. action of a group $\Lambda$ having Haagerup's compact approximation property, e.g. $\Lambda \subset \mathrm{SL}(2, \mathbb{Z})$. If $\theta: L^{\infty}(X) \rtimes \Gamma \simeq\left(L^{\infty}(Y) \rtimes \Lambda\right)^{t}$, then there exists a unitary element $u$ in the target algebra such that $\operatorname{Ad}(u) \circ \theta$ takes $L^{\infty}(X)$ onto $L^{\infty}(Y)^{t}$.

It is easy to check that both the rigidity of a Cartan inclusion $A \subset M$ and the property $\mathrm{H}$ of $M$ relative to $A$ are stable to amplifications. Thus, the class HT of factors with Cartan subalgebras satisfying both conditions is stable to amplifications. By 7.1, given an HT factor $M$ there exists a unique equivalence relation $\mathcal{R}_{M}^{H T}$ associated to its unique (up to conjugacy) HT Cartan subalgebra. Thus, for the factors in this class, all OE invariants for $\mathcal{R}_{M}^{H T}$ are isomorphism invariants for the factor $M$ and $\mathscr{F}\left(\mathcal{R}_{M}^{H T}\right)=\mathscr{F}(M)$. In particular, since Gaboriau showed in ([G00], [G01]) that the equivalence relation of any free ergodic action $\mathbb{F}_{n} \curvearrowright X, 2 \leq n<\infty$, has trivial fundamental group and that for different $n$ 's the $\mathbb{F}_{n}$-actions are OE-inequivalent, we derive:

Corollary 7.3. $1^{\circ}$. If $\Gamma \subset \mathrm{SL}(2, \mathbb{Z})$ is finitely generated nonamenable group then the factor $M=L\left(\mathbb{Z}^{2} \rtimes \Gamma\right)=L^{\infty}\left(\mathbb{T}^{2}\right) \rtimes \Gamma$ has trivial fundamental group, $\mathcal{F}(M)=\{1\}$.

$2^{\circ}$. If $\mathbb{F}_{n} \subset \mathrm{SL}(2, \mathbb{Z})$ is some embedding of the free group with $n$ generators, then the $\mathrm{II}_{1}$ factors $L\left(\mathbb{Z}^{2} \rtimes \mathbb{F}_{n}\right)=L^{\infty}\left(\mathbb{T}^{2}\right) \rtimes \mathbb{F}_{n}$ are mutually non-isomorphic, $2 \leq n \leq \infty$.

Corollary 7.3 gave the first examples of $\mathrm{II}_{1}$ factors with trivial fundamental group ([P01b]). In particular, this solved a longstanding problem of Kadison (Problem 3 in [Ka67]), asking whether there exist $\mathrm{II}_{1}$ factors $M$ with the property that $\mathrm{M}_{n \times n}(M) \not$ $M$ for all $n \geq 2$. By 7.3, the group factor $L G$, arising from the arithmetic group $G=\mathbb{Z}^{2} \rtimes \operatorname{SL}(2, \mathbb{Z})$, does satisfy this property, in fact $\mathcal{F}(L G)=\{1\}$. The corollary also gives the first examples of non-isomorphic group measure space factors associated with actions $\mathbb{F}_{n} \curvearrowright X$ of free groups $\mathbb{F}_{n}$ with different number of generators, $n=$ $2,3, \ldots$ It is an open problem whether $L \mathbb{F}_{n} \not \varkappa L \mathbb{F}_{m}$ for $n \neq m$, despite remarkable progress in the study of free group factors through Voiculescu's free probability theory ([Vo90], [Vo94]). Using these techniques, it was shown in ([R94], [Dy93]) that " $L \mathbb{F}_{n}$, $2 \leq n \leq \infty$, are all non-isomorphic" $\Leftrightarrow$ "two of them are non-isomorphic" $\Leftrightarrow$ “F $\left(L \mathbb{F}_{n}\right) \neq \mathbb{R}_{+}^{*}$ for some $2 \leq n<\infty " \Leftrightarrow$ “F $\left(L \mathbb{F}_{n}\right)=\{1\}$ for all $2 \leq n<\infty "$. The same techniques have led to the proof that $\mathbb{Q} \subset \mathcal{F}\left(L \mathbb{F}_{\infty}\right)$ in ([Vo90]) and finally $\mathcal{F}\left(L \mathbb{F}_{\infty}\right)=\mathbb{R}_{+}^{*}$ in $([\mathrm{R} 94])$.

\section{8. vNE strong rigidity from property (T) and malleability}

We now state rigidity results obtained by using malleability of actions as "deformability property" and the property $(\mathrm{T})$ of the acting group as the "rigidity property", discussed in 6.3. These statements are exactly of the type (3.1). They are the first vNE Strong Rigidity results pertaining to von Neumann algebras. 
The source groups $\Gamma$ considered below are required to be ICC and to have infinite w-normal subgroups $H \subset \Gamma$ with the relative property (T). Examples are all the ICC property (T) groups and the groups $\mathbb{Z}^{2} \rtimes \Gamma_{0}$, with $\Gamma_{0} \subset \mathrm{SL}(2, \mathbb{Z})$ non-amenable (cf. [K67], [Ma82], [Bu91]; see [Va05] for more examples). If $\Gamma$ satisfies the property (e.g. $\Gamma$ ICC with property (T)), then $\Gamma \times H^{\prime}$ satisfies the property as well for all $H^{\prime}$ ICC.

Theorem 8.1 ([P03], [P04a]). Assume $\Gamma$ is ICC and has an infinite normal subgroup with the relative property $(\mathrm{T})$. Let $\Gamma \curvearrowright(X, \mu)$ be an arbitrary free ergodic m.p. action. Let $\Lambda$ be an arbitrary ICC group and $\Lambda \curvearrowright(Y, v)$ a Bernoulli action, or more generally a free, relative weak mixing quotient of a Bernoulli $\Lambda$-action.

If $\theta: L^{\infty}(X) \rtimes \Gamma \simeq\left(L^{\infty}(Y) \rtimes \Lambda\right)^{t}$ is an isomorphism of $\mathrm{II}_{1}$ factors, for some $0<t \leq 1$, then $t=1$ and $\theta$ is of the form $\theta=\operatorname{Ad}(u) \circ \theta^{\gamma} \circ \theta^{\Delta, \delta}$, where: $u$ is a unitary element in $L^{\infty}(Y) \rtimes \Lambda ; \theta^{\gamma} \in \operatorname{Aut}\left(L^{\infty}(Y) \rtimes \Lambda\right)$ is implemented by some $\gamma \in \operatorname{Hom}(\Lambda, \mathbb{T}) ; \theta^{\Delta, \delta}: L^{\infty}(X) \rtimes \Gamma \simeq L^{\infty}(Y) \rtimes \Lambda$ is implemented by isomorphisms $\Delta:(X, \mu) \simeq(Y, v), \delta: \Gamma \simeq \Lambda$ which give a conjugacy of $\Gamma \curvearrowright X, \Lambda \curvearrowright Y$.

More generally, the above statement holds true for target actions $\Lambda \curvearrowright Y$ that are relative weak mixing quotients of actions that are s-malleable and clustering. An action $\Lambda \curvearrowright(Y, v)$ is a quotient of an action $\Lambda \curvearrowright\left(Y^{\prime}, v^{\prime}\right)$ if there exists a $\Lambda$ invariant m.p. surjection $\left(Y^{\prime}, v^{\prime}\right) \rightarrow(Y, v)$, equivalently a $\Lambda$-invariant m.p. embed$\operatorname{ding} L^{\infty}(Y) \subset L^{\infty}\left(Y^{\prime}\right)$. The quotient is relative weak mixing if there exists $g_{n} \rightarrow \infty$ in $\Lambda$ such that $\lim _{n}\left\|E_{L^{\infty}(Y)}\left(x g_{n}(y)\right)\right\|_{2}=0$ for all $x, y \in L^{\infty}\left(Y^{\prime}\right)$, with $y \perp L^{\infty}(Y)$ (cf. Furstenberg and Zimmer). The clustering condition is a certain multi-mixing condition which is satisfied, like s-malleability, by all Bernoulli actions.

The proof of this result is in three parts: The malleability of $\Lambda \curvearrowright Y$ combined with the rigidity assumption on $\Gamma$ allows a deformation/rigidity argument in $\tilde{M}=$ $L^{\infty}(Y \times Y) \rtimes \Lambda$. Taking $t=1, M=L^{\infty}(Y) \rtimes \Lambda=L^{\infty}(X) \rtimes \Gamma$ for simplicity, this gives rise to a non-zero $L \Gamma-L \Lambda$ Hilbert bimodule $\mathscr{H} \subset L^{2}(M)$ which is finite dimensional as a right $L \Lambda$-module. Using intertwining technique, from $\mathscr{H}$ one gets a unitary element $u \in M$ that conjugates $L \Gamma$ into $L \Lambda$. The second part of the proof uses this information to derive that $L^{\infty}(X)$ can be unitarily conjugated onto $L^{\infty}(Y)$, by using ultrapower algebra techniques, "asymptotic analysis" of Fourier expansions and 5.1 (repeatedly). The final part of the proof consists in showing that if both the Cartan subalgebras and the group algebras can be (separately) conjugated by unitaries, then there exists a unitary that conjugates $L^{\infty}(X)$ onto $L^{\infty}(Y)$ and carries the canonical unitaries of the source factor onto scalar multiples of the canonical unitaries of the target factor.

It has been recently shown in ([P06]) that in fact 8.1 holds true for any group of the form $\Gamma=H H^{\prime}$ with $H$ non-amenable and $H^{\prime}$ infinite (e.g. $\Gamma$ non-amenable with infinite center, or $\Gamma=H \times H^{\prime}$ ), and even for groups $\Gamma$ that have a non-amenable subgroup $H$ whose centralizer is infinite and w-normal in $\Gamma$.

Taking the source and target group actions to satisfy both sets of conditions, and taking into account that a factor arising from the Bernoulli $\Gamma$-action with diffuse base 
is the same as the group factor of the wreath product $\mathbb{Z} \imath \Gamma$, from 8.1 we derive a positive answer to a wreath product version of Connes' rigidity conjecture (3.2):

Corollary 8.2. Let $\Gamma_{i}$ be an ICC group having an infinite normal subgroup with the relative property $(\mathrm{T})$ and denote $G_{i}=\mathbb{Z} 2 \Gamma_{i}$ the corresponding wreath product, $i=0,1$. Then $L G_{0} \simeq L G_{1}^{t}$ implies $t=1, G_{0} \simeq G_{1}$ and $\Gamma_{0} \simeq \Gamma_{1}$. In particular, all such factors have trivial fundamental group.

We mention that (6.4 and 7.13 in [PV06]) provides a class $\mathcal{W}$ of "generalized" wreath product groups for which a $\left(3.2^{\prime}\right)$-type statement (i.e. a version of the strong Connes rigidity conjecture) holds true, i.e. any isomorphism $\theta: L G_{1} \simeq L G_{2}^{t}$, $G_{1}, G_{2} \in \mathcal{W}$, entails $t=1$ and $\theta=\operatorname{Ad}(u) \circ \theta^{\gamma} \circ \theta^{\delta}$, for some $\gamma \in \operatorname{Hom}\left(G_{2}, \mathbb{T}\right)$ and $\delta: G_{1} \simeq G_{2}$. In particular, if $G \in \mathcal{W}$ then $\operatorname{Out}(L G)=\operatorname{Hom}(G, \mathbb{T}) \rtimes \operatorname{Out}(G)$.

Arguments similar to the ones used in the first part of the proof of 8.1 allow showing that for any countable subgroup $S \subset \mathbb{R}_{+}^{*}$ there exist $\mathrm{II}_{1}$ factors and equivalence relations from ergodic m.p. actions of countable groups which have $S$ as fundamental group.

Theorem 8.3 ([P03]). Let $S \subset \mathbb{R}_{+}^{*}$ be a countable subgroup and $\left\{s_{n}\right\}_{n} \subset S$ a set of generators. Let $\Gamma$ be an ICC group having an infinite w-normal subgroup with the relative property $(\mathrm{T})$. For each $n$, let $\mu_{n}$ be the probability measure on $\{0,1\}$ satisfying $\mu_{n}(\{0\}) / \mu_{n}(\{1\})=s_{n}$ and let $s_{n}$ be the equivalence relation on $\left(\{0,1\}, \mu_{n}\right)^{\Gamma}$ given by $\left(t_{g}\right)_{g} \sim\left(t_{g}^{\prime}\right)_{g}$ iff there exists a finite subset $F \subset \Gamma$ such that $t_{g}=t_{g}^{\prime}$ for all $g \in \Gamma \backslash F$ and $\Pi_{g \in F} \mu_{0}\left(t_{g}\right)=\Pi_{g \in F} \mu_{0}\left(t_{g}^{\prime}\right)$. Let $\mathcal{R}_{0}$ be the product of the equivalence relations $\S_{n}$ on the product probability space $(X, \mu)=\Pi_{n}\left(\{0,1\}, \mu_{n}\right)^{\Gamma}$ and $\mathcal{R}$ be the equivalence relation generated by $\mathcal{R}_{0}$ and the Bernoulli $\Gamma$-action $\Gamma \curvearrowright X$ (which leaves $\mathcal{R}_{0}$ invariant). If $\mathcal{F}(L \Gamma)=\{1\}$, for instance if $\Gamma=H \rtimes \Gamma_{0}$ with $\Gamma_{0} \subset \operatorname{SL}(2, \mathbb{Z})$ finite index subgroup $(c f .7 .3)$, then $\mathcal{F}(L(\mathcal{R}))=\mathcal{F}(\mathcal{R})=S$.

A construction similar to 8.3 can be used to prove that in fact any subgroup $S \subset \mathbb{R}_{+}^{*}$ can be realized as the fundamental group of a non-separable $\mathrm{II}_{1}$ factor ([P03]), solving completely a problem raised by Murray-von Neumann in ([MvN43]). This construction of concrete equivalence relations $\mathcal{R}$ with arbitrary countable fundamental group, obtained through $\mathrm{II}_{1}$ factor methods, is completely new for OE theory as well.

When applied to isomorphisms $\theta$ coming from an $\mathrm{OE} \mathcal{R}_{\Gamma} \simeq\left(\mathcal{R}_{\Lambda}\right)^{t}, 8.1$ becomes an $\mathrm{OE}$ strong rigidity result. In fact, one can even derive an OE Strong Rigidity for embeddings of equivalence relations:

Theorem 8.4 ([P04a]). Let $\Gamma \curvearrowright X, \Lambda \curvearrowright Y$ be as in 8.1. If $\Delta:(X, \mu) \simeq(Y, v)$ takes each $\Gamma$-orbit into a $\Lambda$-orbit then there exist a subgroup $\Lambda_{0} \subset \Lambda$ and $\alpha \in[\Lambda]$ such that $\alpha \circ \Delta$ conjugates $\Gamma \curvearrowright X, \Lambda_{0} \curvearrowright Y$. 


\section{Cocycle superrigidity from property (T) and malleability}

If in Theorem 8.4 we take $\Gamma$ to have property (T) and $\Delta$ to give an orbit equivalence of $\Gamma \curvearrowright X, \Lambda \curvearrowright Y$, and use that property (T) is an OE invariant, then the statement becomes an OE Strong Rigidity where all conditions are on one side, a type of result labelled "OE superrigidity" in Section 3. In fact, if we assume all conditions are say on the side of the source group action $\Gamma \curvearrowright X$, then the deformation/rigidity arguments in the proof of 8.1 get simplified considerably, allowing us to prove a cocycle superrigidity result from which the $\mathrm{OE}$ superrigidity is a mere consequence.

To state the result, recall that if $\mathcal{V}$ is a Polish group then a $\mathcal{V}$-valued measurable (left) cocycle for $\Gamma \curvearrowright X$ is a measurable map $w: \Gamma \times X \rightarrow \mathcal{V}$ satisfying $w\left(g_{1}, g_{2} t\right) w\left(g_{2}, t\right)=w\left(g_{1} g_{2}, t\right)$ for all $g_{1}, g_{2} \in \Gamma, t \in X$. Cocycles $w, w^{\prime}$ are equivalent if there is a measurable $u: X \rightarrow \mathcal{V}$ such that $w^{\prime}(g, t)=u(g t) w(g, t) u(t)^{-1}$ for all $g, t$ (a.e.). Note that $w$ is independent of $t \in X$ iff it is a group morphism $\Gamma \rightarrow \mathcal{V}$.

A Polish group $\mathcal{V}$ is of finite type if it is isomorphic to a closed subgroup of the group of unitary elements $U(N)$ of a finite von Neumann algebra $N$ (equivalently of a II 1 factor). We denote by $U_{\text {fin }}$ the class of Polish groups of finite type. All countable discrete groups and all separable compact groups are of finite type. But by ([KaSi82]) a connected locally compact group $\mathcal{V}$ is of finite type iff $\mathcal{V}=K \times V$ with $K$ compact and $V \simeq \mathbb{R}^{n}$.

Theorem 9.1 (P05]). Assume $\Gamma$ has an infinite $w$-normal subgroup $H$ with the relative property $(\mathrm{T})$ and that $\Gamma \curvearrowright(X, \mu)=\left(X_{0}, \mu_{0}\right)^{I}$ is a generalized Bernoulli action with $|H i|=\infty$ for all $i \in I$.

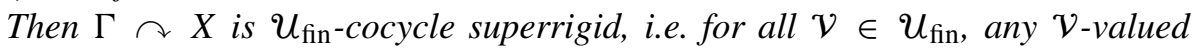
cocycle for $\Gamma \curvearrowright X$ is equivalent to a group morphism $\Gamma \rightarrow \mathcal{V}$. Moreover, same is true if $\Gamma \curvearrowright X$ is a relative weak mixing quotient of an action satisfying these conditions.

The above statement actually holds true for all malleable actions which are weak mixing on the subgroup $H$, and for all relative weak mixing quotients of such actions. The proof uses a version of the general deformation/rigidity argument explained in 6.2, in the group measure space von Neumann algebra $M=L^{\infty}(X) \bar{\otimes} \rtimes \Gamma$, where $V$ is a $\mathrm{II}_{1}$ factor with $\mathcal{V}=\overline{\mathcal{V}} \subset \mathcal{U}(V)$ and $\Gamma \curvearrowright L^{\infty}(X) \bar{\otimes} V$ is the product of the action $\Gamma \curvearrowright L^{\infty}(X)$ and the trivial action of $\Gamma$ on $V$. Also, the larger algebra $\tilde{M}$ in which we perform the deformation is $\left(L^{\infty}(X) \bar{\otimes} V \bar{\otimes} L^{\infty}(X)\right) \rtimes \Gamma$. We use the observation that a measurable function $w: \Gamma \times X \rightarrow \mathcal{U}(V)$ is a cocycle for $\Gamma \curvearrowright X$ iff the $g \mapsto u_{g}^{\prime}=w_{g} u_{g}$ is a representation of the group $\Gamma$ in $M$, where $u_{g}$ denote the canonical unitaries in $M$, and $w_{g}=w(g, \cdot)$. With the notations in 6.2, the algebra $N$ corresponds to $L \Gamma \bar{\otimes} V$ while $P$ corresponds to the von Neumann algebra generated by $u_{g}^{\prime}, g \in \Gamma$. Due to the more concrete form of this set-up, the deformation/rigidity argument in $\tilde{M}$ gives in fact an intertwiner of the $\Gamma$-representations $u_{g}^{\prime}$ and $\alpha_{1}\left(u_{g}^{\prime}\right)$, which lies in $L^{\infty}(X) \bar{\otimes} \nabla \bar{\otimes} L^{\infty}(X)$. After the usual "re-interpretation" of this intertwiner, we get $u \in U\left(L^{\infty}(X) \otimes V\right)$ such that $u u_{h}^{\prime} u^{*} \in 1 \otimes V \rtimes \Gamma$. This means 
$u w_{h} h(u)^{*} \in V$, so we have untwisted $w$ as a $U(V)$-valued cocycle for $H \curvearrowright X$. But then it follows automatically untwisted as a $\mathcal{V}$-valued cocycle, due to the weak mixing property. Applying the w-normality of $H \subset \Gamma$ and the weak mixing property again, it follows untwisted on all $\Gamma$.

The $\mathrm{II}_{1}$ factor framework is used again to show that if a group action $\Gamma \curvearrowright X$ is cocycle superrigid then it is $\mathrm{OE}$ superrigid, i.e. any $\mathrm{OE} \Delta: X \simeq Y$ of this action with an arbitrary free action $\Lambda \curvearrowright Y$ comes from a conjugacy. More precisely, let $w=w_{\Delta}: \Gamma \times X \rightarrow \Lambda$ be the Zimmer cocycle associated to $\Delta$, which to $t \in X$, $g \in \Gamma$ assigns the unique (by freeness) $h \in \Lambda$ such that $\Delta(g t)=h \Delta(t)$ ([Z80], [Z84]). Since $w$ takes values in $\Lambda$, which is discrete and thus in the class $U_{\text {fin }}$, by 9.1 it can be untwisted. The $\mathrm{II}_{1}$ factor setting allows to re-interpret the "untwister" of this cocycle as a natural "conjugator" of the two actions, thus showing that cocycle superrigidity with arbitrary discrete targets implies OE superrigidity:

Theorem 9.2 ([P05]). Let $\Gamma \curvearrowright X$ be a free, weakly mixing, cocycle superrigid action, e.g. an action as in 9.1. Assume $\Gamma$ has no finite normal subgroups. Let $\Lambda \curvearrowright(Y, v)$ be an arbitrary free ergodic m.p. action and $\Delta: \mathcal{R}_{\Gamma} \simeq \mathcal{R}_{\Lambda}^{t}$ an orbit equivalence, for some $t>0$.

Then $n=1 / t$ is an integer and there exist a subgroup $\Lambda_{0} \subset \Lambda$ of index $\left[\Lambda: \Lambda_{0}\right]=$ $n$, a subset $Y_{0} \subset Y$ of measure $v\left(Y_{0}\right)=1 / n$ fixed by $\Lambda_{0} \curvearrowright Y$, an automorphism $\alpha \in[\Lambda]$ and a group isomorphism $\delta: \Gamma \simeq \Lambda_{0}$ such that $\alpha \circ \Delta$ takes $X$ onto $Y_{0}$ and conjugates the actions $\Gamma \curvearrowright X, \Lambda_{0} \curvearrowright Y_{0}$.

In fact, 9.1 even implies the superrigidity of embeddings of $\mathcal{R}_{\Gamma}$ into equivalence relations $\mathcal{R}_{\Lambda}$ of arbitrary free ergodic actions (in the spirit of 8.4), as well as for morphisms of $\mathcal{R}_{\Gamma}$ onto $\mathcal{R}_{\Lambda}$ that are bijective on each orbit, i.e. for local $O E$ morphisms (see [P05] for details). Theorem 9.1 also shows that the $\mathbb{T}$-valued first cohomology group $\mathrm{H}^{1}\left(\mathcal{R}_{\Gamma}\right)$ of a Bernoulli $\Gamma$-action is equal to $\operatorname{Hom}(\Gamma, \mathbb{T})$, thus recovering a result from ([P01a], [PSa03]). This implies that given any abelian group $L$ one can construct free quotients of Bernoulli $\Gamma$-actions with first cohomology group $\mathrm{H}^{1}\left(\mathcal{R}_{\Gamma}\right)=\operatorname{Hom}(\Gamma, \mathbb{T}) \times L$ (cf. [P04b]). Thus, any group $\Gamma$ with an infinite w-normal subgroup with the relative property $(\mathrm{T})$ has uncountably many "concrete" non-OE free ergodic m.p. actions. Another application of 9.1 shows that the equivalence relations $\mathcal{R}$ described in the statement of 8.3 have the property that for all $t>0, \mathcal{R}^{t}$ cannot be implemented by a free ergodic action of a group (5.10 in [P05]). The first examples of equivalence relations with this property were found in ([Fu99b]).

The Cocycle Superrigidity 9.1 was recently used in ([Fu06]) to show that if $\Gamma, \Lambda$ are lattices in a higher rank semisimple Lie group $g$ then the action $\Gamma \curvearrowright g / \Lambda$ cannot be realized as a quotient of a Bernoulli $\Gamma$-action, more generally of a s-malleable weak mixing action. Also, 9.1 was used in ([Th06]) to answer some open problems in descriptive set theory, showing for instance that the universal countable Borel equivalence relation $E_{\infty}$ cannot be implemented by a free action of a countable

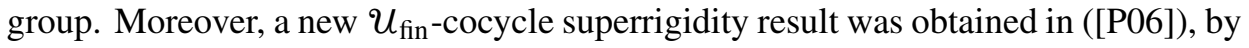
combining malleability with spectral gap rigidity (see final comment in 6.6 above). 
It shows that if $\Gamma \curvearrowright X$ is a generalized Bernoulli action and $H \subset \Gamma$ is a subgroup such that $H \curvearrowright X$ has spectral gap (thus $H$ is automatically non-amenable) then any $\mathcal{V}$-valued cocycle for $\Gamma \curvearrowright X$, where $\mathcal{V} \in U_{\text {fin }}$, can be untwisted on the centralizer $H^{\prime}$ of $H$ in $\Gamma$. Thus, if $H^{\prime}$ is w-normal in $\Gamma$ (e.g. $\Gamma=H \times H^{\prime}$ ) and $H^{\prime} \curvearrowright X$ is weak mixing then the cocycle follows untwisted on all $\Gamma$. In particular, such $\Gamma \curvearrowright X$ are OE superrigid. This adds to the rigidity phenomena involving product groups discovered in recent years in ergodic theory, Borel equivalence relations and $\mathrm{II}_{1}$ factors (e.g. [MoS02], [MoS04], [OzP04], [HjKe05]).

\section{Bass-Serre type rigidity for amalgamated free products}

The malleable deformation explained in the second part of 6.4 is used in ([IPeP05]) to prove a series of rigidity results, through the deformation/rigidity approach. These results can be viewed as von Neumann algebra versions of the "subgroup theorems" and "isomorphism theorems" for amalgamated free products of groups in Bass-Serre theory. The main "subalgebra theorem" shows that, under rather general conditions, any rigid von Neumann subalgebra $Q \subset M=M_{1} *_{B} M_{2}$ can be conjugated by an inner automorphism of $M$ into either $M_{1}$ or $M_{2}$. For simplicity, we only formulate the result in the case $M=\left(B \rtimes \Gamma_{1}\right) *_{B}\left(B \rtimes \Gamma_{2}\right)=B \rtimes\left(\Gamma_{1} * \Gamma_{2}\right)$.

Theorem 10.1 ([IPeP05]). Let $\Gamma \curvearrowright(B, \tau)$ be an action of a group $\Gamma=\Gamma_{1} * \Gamma_{2}$ on a finite von Neumann algebra $(B, \tau)$ and denote $M_{i}=B \rtimes \Gamma_{i}, i=1,2, M=$ $B \rtimes \Gamma=M_{1} *_{B} M_{2}$. Let $Q \subset M$ be a rigid inclusion. Assume no corner $q Q q$ of $Q$ can be embedded into $B$ and that the normalizer of $Q$ in $M$ generates a factor $P$. Then there exists a unique $i \in\{1,2\}$ such that $u Q u^{*} \subset M_{i}$ for some $u \in \mathcal{U}(M)$, which also satisfies $u P u^{*} \subset M_{i}$.

Taking the action $\Gamma \curvearrowright B$ in 10.1 to come from an action on a probability space, the theorem can be used to prove Bass-Serre type vNE and OE rigidity results for actions of free products of groups, as follows:

Theorem 10.2 ([IPeP05]). Let $\Gamma_{i}, \Lambda_{j}, 1 \leq i \leq n \leq \infty, 1 \leq j \leq m \leq \infty$, be ICC groups having normal, non virtually abelian subgroups with the relative property $(\mathrm{T})$. Denote $\Gamma=\Gamma_{1} * \cdots, \Lambda=\Lambda_{1} * \cdots$ and let $\Gamma \curvearrowright(X, \mu), \Lambda \curvearrowright(Y, v)$ be free m.p. actions, ergodic oneach $\Gamma_{i}, \Lambda_{j}, i, j \geq 1$. Denote $M=L^{\infty}(X) \rtimes \Gamma, N=L^{\infty}(Y) \rtimes \Lambda$, $M_{i}=L^{\infty}(X) \rtimes \Gamma_{i} \subset M, N_{j}=L^{\infty}(Y) \rtimes \Lambda_{j} \subset N$ the corresponding group measure space factors. If $\theta: M \simeq N^{t}$ is an isomorphism, for some $t>0$, then $m=n$ and there exists a permutation $\pi$ of indices $j \geq 1$ and unitaries $u_{j} \in M_{2}^{t}$ such that $\operatorname{Ad}\left(u_{j}\right)\left(\theta\left(M_{j}\right)\right)=N_{\pi(j)}^{t}, \operatorname{Ad}\left(u_{j}\right)\left(\theta\left(L^{\infty}(X)\right)\right)=\left(L^{\infty}(Y)\right)^{t}$ for all $j \geq 1$. In

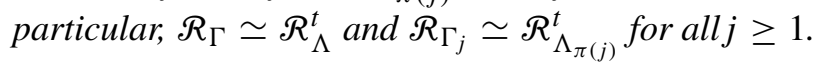

In particular, taking the isomorphism $\theta$ between the group measure space factors in 10.2 to come from an orbit equivalence of the actions, one gets the following converse to a result in ([G05]): 
Corollary 10.3. Let $\Gamma_{i}, \Lambda_{j}, 1 \leq i \leq n \leq \infty, 1 \leq j \leq m \leq \infty, \Gamma \curvearrowright(X, \mu)$, $\Lambda \curvearrowright(Y, v)$ be as in 10.2. If $\mathcal{R}_{\Gamma} \simeq \mathcal{R}_{\Lambda}^{t}$ then $n=m$ and there exists a permutation $\pi$ of the set of indices $i \geq 1$ such that $\mathcal{R}_{\Gamma_{i}} \simeq \mathcal{R}_{\Lambda_{\pi(i)}}^{t}$ for all $i \geq 1$.

In turn, applying 10.1 to $\Gamma$-actions on the hyperfinite $\mathrm{II}_{1}$ factor $B=R$ and using results from ([P90]) one can prove:

Theorem 10.4 ([IPeP05]). $1^{\circ}$. For any $\Gamma_{0}=\mathrm{SL}\left(n_{0}, \mathbb{Z}\right), n_{0} \geq 2$ and any group $\Gamma_{1}$ having an infinite normal subgroup with the relative property $(\mathrm{T})$ there exist properly outer actions $\Gamma_{0} * \Gamma_{1} \curvearrowright R$ such that:

(a) $R \subset R \rtimes \Gamma_{0}$ is a rigid inclusion;

(b) $\Gamma_{1} \curvearrowright R$ is a non-commutative Bernoulli action, i.e. $R$ can be represented as $R=\bar{\otimes}_{g \in \Gamma_{1}}\left(\mathrm{M}_{n \times n}(\mathbb{C}), \operatorname{tr}\right)_{g}, n \geq 2$, with $\Gamma_{1}$ acting on it by Bernoulli shifts;

(c) $\Gamma_{1} \subset \operatorname{Out}(R)$ is freely independent with respect to the normalizer $\mathcal{N}_{0}$ of $\Gamma_{0}$ in $\operatorname{Out}(R)$.

$2^{\circ}$. If $\Gamma_{0} * \Gamma_{1} \curvearrowright R$ is an action as in $1^{\circ}$ and $M=R \rtimes\left(\Gamma_{0} * \Gamma_{1}\right)$, then $\mathcal{F}(M)=\{1\}$ and $\operatorname{Out}(M)=\operatorname{Hom}\left(\Gamma_{0}, \mathbb{T}\right) \times \operatorname{Hom}\left(\Gamma_{1}, \mathbb{T}\right)$. In particular, given any separable compact abelian group $K$, there exist free actions of $\Gamma=\operatorname{SL}(3, \mathbb{Z}) *(\operatorname{SL}(3, \mathbb{Z}) \times \hat{K})$ on $R$ such that $M=R \rtimes \Gamma$ satisfies $\mathcal{F}(M)=\{1\}$ and $\operatorname{Out}(M)=K$.

Part $2^{\circ}$ of the above theorem gives the first examples of $\mathrm{II}_{1}$ factors with calculable outer automorphism group, in particular of factors with trivial outer automorphism group, thus answering in the affirmative a well known problem posed by Connes in 1973. However, since the proof of part $1^{\circ}$ uses a Baire category argument, $10.4 .2^{\circ}$ is actually an existence result. By using a refinement of techniques in ([P03], [P04a]) and results from ([Oz04]), some concrete examples of group measure space factors with trivial outer automorphism group were recently constructed in ([PV06]) from generalized Bernoulli actions of $\Gamma=\operatorname{SL}(4, \mathbb{Z}) \ltimes \mathbb{Z}^{4}$ on $(X, \mu)=\left(\{0,1\}, \mu_{0}\right)^{\Gamma / \Gamma_{0}}$, with $\Gamma_{0}$ a certain abelian subgroup of $\Gamma$ and $\mu_{0}(0) \neq \mu_{0}(1)$. In fact, ([PV06]) gives also concrete examples of factors $M$ with $\operatorname{Out}(M)$ any prescribed finitely generated group.

Note that when applied to the case $B=\mathbb{C}, 10.1$ becomes a von Neumann algebra analogue of Kurosh's classical theorem for free products of groups, similar to Ozawa's pioneering result of this type in ([Oz04]; see also his paper in these proceedings), but covering a different class of factors and allowing amplifications. For instance, if $M_{i}=L \Gamma_{i}, 2 \leq i \leq n, L \Lambda_{j}, 2 \leq j \leq m$, are factors from ICC groups having infinite normal subgroups with relative property (T), then $M_{1} * M_{2} * \cdots * M_{m} \stackrel{\theta}{\simeq}$ $\left(N_{1} * N_{2} * \cdots * N_{n}\right)^{t}$ implies $m=n$ and $\theta\left(M_{i}\right)$ inner conjugate to $N_{i}^{t}$ for all $i$, after some permutation of indices. When combined with results in ([DyR00]) and to the fact that $P=L\left(\mathbb{Z}^{2} \rtimes \mathrm{SL}(2, \mathbb{Z})\right)$ has trivial fundamental group ([P01b]), this can be used to show that for all subgroups $S=\left\{s_{j}\right\}_{j} \subset \mathbb{R}_{+}^{*}, M=*_{j} P^{s_{j}}$ has fundamental 
group equal to $S$. This provides a completely new class of factors $M$ with $\mathcal{F}(M)$ an arbitrary subgroup $S \subset \mathbb{R}_{+}^{*}$, than the ones in ([P03]). Indeed, by Voiculescu's striking result in ([V94], cf. also [Sh04]) the factors $*_{j} P^{s_{j}}$ have no Cartan subalgebras, while the ones in ([P03]) arise from equivalence relations.

\section{References}

[An87] Anantharaman-Delaroche, C., On Connes' property (T) for von Neumann algebras. Math. Japon. 32 (1987), 337-355.

[Bo93] Boca, F., On the method for constructing irreducible finite index subfactors of Popa. Pacific J. Math. 161 (1993), 201-231.

[Bu91] Burger, M., Kazhdan constants for SL(3, Z). J. Reine Angew. Math. 413 (1991), 36-67.

[CaH85] de Cannière, J., Haagerup, U., Multipliers of the Fourier algebra of some simple Lie groups and their discrete subgroups. Amer. J. Math. 107 (1985), 455-500.

[CCJJV01] Cherix, P.-A., Cowling, M., Jolissaint, P., Julg, P., Valette, A., Groups with Haagerup property. Progr. Math. 197, Birkhäuser, Basel 2001.

[Cho83] Choda, M., Group factors of the Haagerup type. Proc. Japan Acad. 59 (1983), 174-177.

[Ch79] Christensen, E., Subalgebras of a finite algebra. Math. Ann. 243 (1979), 17-29.

[C75] Connes, A., Sur la classification des facteurs de type II. C. R. Acad. Sci. Paris 281 (1975), 13-15.

[C76] Connes, A., Classification of injective factors. Ann. of Math. 104 (1976), 73-115.

[C80] Connes, A., A type $\mathrm{II}_{1}$ factor with countable fundamental group. J. Operator Theory 4 (1980), 151-153.

[C82] Connes, A., Classification des facteurs. In Operator algebras and applications (Kingston, Ont., 1980), Part 2, Proc. Sympos. Pure Math. 38, Amer. Math. Soc., Providence, R.I., 1982, 43-109.

[CFW81] Connes, A., Feldman, J., Weiss, B., An amenable equivalence relation is generated by a single transformation. Ergodic Theory Dynam. Systems 1 (1981), 431-450.

[CJ82] Connes, A., Jones, V. F. R., A II 1 factor with two non-conjugate Cartan subalgebras. Bull. Amer. Math. Soc. 6 (1982), 211-212.

[CJ85] Connes, A., Jones, V. F. R., Property (T) for von Neumann algebras. Bull. London Math. Soc. 17 (1985), 57-62.

[CSh03] Connes, A., Shlyakhtenko, D., $\mathrm{L}^{2}$-homology for von Neumann algebras. J. Reine Angew. Math. 586 (2005), 125-168.

[CW80] Connes, A., Weiss, B., Property (T) and asymptotically invariant sequences. Israel J. Math. 37 (1980), 209-210.

[CoH89] Cowling, M., Haagerup, U., Completely bounded multipliers and the Fourier algebra of a simple Lie group of real rank one. Invent. Math. 96 (1989), 507-549.

[CoZ89] Cowling, M., Zimmer, R., Actions of lattices in $\operatorname{Sp}(n, 1)$. Ergodic Theory Dynam. Systems 9 (1989), 221-237. 
[Di54] Dixmier, J., Sous-anneaux abéliens maximaux dans les facteurs de type fini. Ann. of Math. 59 (1954), 279-286.

[D59] Dye, H., On groups of measure preserving transformations I. Amer. J. Math. 81 (1959), 119-159.

[D63] Dye, H., On groups of measure preserving transformations II. Amer. J. Math. 85 (1963), 551-576.

[Dy93] Dykema, K., Interpolated free group factors. Duke Math J. 69 (1993), 97-119.

[DyR00] Dykema, K., Rădulescu, F., Compressions of free products of von Neumann algebras. Math. Ann. 316 (1) (2000), 61-82.

[EL77] Effros, E., Lance, C., Tensor products of operator algebras. Adv. Math. 25 (1977), $1-34$.

[FM77] Feldman, J., Moore, C. C., Ergodic equivalence relations, cohomology, and von Neumann algebras I; II. Trans. Amer. Math. Soc. 234 (1977), 289-324; 325-359.

[Fu99a] Furman, A., Gromov's measure equivalence and rigidity of higher rank lattices. Ann. of Math. 150 (1999), 1059-1081.

[Fu99b] Furman, A., Orbit equivalence rigidity. Ann. of Math. 150 (1999), 1083-1108.

[Fu06] Furman, A., On Popa's Cocycle Superrigidity Theorem. In preparation.

[G00] Gaboriau, D., Cout des rélations d'équivalence et des groupes. Invent. Math. 139 (2000), 41-98.

[G01] Gaboriau, D., Invariants $\ell^{2}$ de rélations d'équivalence et de groupes. Inst. Hautes Études Sci. Publ. Math. 95 (2002), 93-150.

[G05] Gaboriau, D., Examples of groups that are measure equivalent to free groups. Ergodic Theory Dynam. Systems 25 (6) (2005), 1809-1827.

[GP03] Gaboriau, D., Popa, S., An Uncountable Family of Non Orbit Equivalent Actions of $\mathbb{F}_{n}$. J. Amer. Math. Soc. 18 (2005), 547-559.

[GeGo88] Gefter, S. L., Golodets, V. Y., Fundamental groups for ergodic actions and actions with unit fundamental groups. Publ. Res. Inst. Math. Sci. 6 (1988), 821-847.

[GoNe87] Golodets, V. Y., Nesonov, N. I., T-property and nonisomorphic factors of type II and III. J. Funct. Anal. 70 (1987), 80-89.

[HaV89] de la Harpe, P., Valette, A., La propriété T de Kazhdan pour les groupes localement compacts. Astérisque 175, (1989).

[H79] Haagerup, U., An example of a non-nuclear $C^{*}$-algebra which has the metric approximation property. Invent. Math. 50 (1979), 279-293.

[Hj02] Hjorth, G., A converse to Dye's Theorem. Trans Amer. Math. Soc. 357 (2004), 3083-3103.

[HjKe05] Hjorth, G., Kechris, A., Rigidity theorems for actions of product groups and countable Borel equivalence relations. Mem. Amer. Math. Soc. 177 (833) 2005.

[I04] Ioana, A., A relative version of Connes $\chi(M)$ invariant. Ergodic Theory Dynam. Systems, to appear; math.OA/0411164.

[IPeP05] Ioana, A., Peterson, J., Popa, S., Amalgamated free products of w-rigid factors and calculation of their symmetry groups. Acta Math., to appear; math.OA/0505589.

[Jo02] Jolissaint, P., On Property (T) for pairs of topological groups. L'Enseignement Math. 51 (2005), 31-45. 
[J83] Jones, V. F. R., Index for subfactors. Invent. Math. 72 (1983), 1-25.

[J90] Jones, V. F. R., von Neumann algebras in mathematics and physics. In Proceedings of the International Congress of Mathematicians (Kyoto, 1990), Vol. I, The Mathematical Society of Japan, Tokyo, Springer-Verlag, Tokyo, 1991, 121-138.

[J00] Jones, V. F. R., Ten problems. In Mathematics: perspectives and frontieres (ed. by V. Arnold, M. Atiyah, P. Lax and B. Mazur), Amer. Math. Soc., Providence, RI, 2000, 79-91.

[Ka67] Kadison, R. V., Problems on von Neumann algebras. Baton Rouge Conference 1967.

[KaSi52] Kadison, R. V., Singer, I. M., Some remarks on representations of connected groups. Proc. Nat. Acad. Sci. U.S.A. 38 (1952), 419-423.

[K67] Kazhdan, D., Connection of the dual space of a group with the structure of its closed subgroups. Funct. Anal. Appl. 1 (1967), 63-65.

[Ma82] Margulis, G., Finitely-additive invariant measures on Euclidian spaces. Ergodic Theory Dynam. Systems 2 (1982), 383-396.

[Mc70] McDuff, D., Central sequences and the hyperfinite factor. Proc. London Math. Soc. 21 (1970), 443-461.

[Mo06] Monod, N., An invitation to bounded cohomology. In Proceedings of the International Congress of Mathematicians (Madrid, 2006), Vol. II, EMS Publishing House, Zürich 2006, 1183-1211.

[MoS02] Monod, N., Shalom, Y., Orbit equivalence rigidity and bounded cohomology. Ann. of Math. 164 (2006), 825-878.

[MoS04] Monod, N., Shalom, Y., Cocycle superrigidity and bounded cohomology for negatively curved spaces. J. Differential Geom. 67 (2004), 395-455.

[M82] Moore, C. C., Ergodic theory and von Neumann algebras. In Operator algebras and applications (Kingston, Ont., 1980), Part 2, Proc. Symp. Pure Math. 38, Amer. Math. Soc., Providence, R.I., 179-226.

[MvN36] Murray, F., von Neumann, J., On rings of operators. Ann. of Math. 37 (1936), 116-229.

[MvN43] Murray, F., von Neumann, J., Rings of operators IV.Ann. of Math. 44 (1943), 716-808.

[OW80] Ornstein, D., Weiss, B., Ergodic theory of amenable group actions I. The Rohlin Lemma. Bull. Amer. Math. Soc. (1) 2 (1980), 161-164.

[Oz02] Ozawa, N., There is no separable universal $\mathrm{II}_{1}$-factor. Proc. Amer. Math. Soc. 132 (2004), 487-490.

[Oz04] Ozawa, N., A Kurosh type theorem for type $\mathrm{II}_{1}$ factors. Internat. Math. Res. Notices, Article ID 97560.

[OzP04] Ozawa, N., Popa, S., Some prime factorization results for type $\mathrm{II}_{1}$ factors. Invent Math. 156 (2004), 223-234.

[Pe04] Peterson, J., A 1-cohomology characterization of property (T) in von Neumann algebras. Pacific J. Math., to appear; math.OA/0409527.

[PeP04] Peterson, J., Popa, S., On the notion of relative property (T) for inclusions of von Neumann algebras. J. Funct. Anal. 219 (2005), 469-483.

[PiP86] Pimsner, M., Popa, S., Entropy and index for subfactors. Ann. Sci. École Norm. Sup. 19 (1986), 57-106. 
[P83] Popa, S., Orthogonal pairs of $*$-subalgebras in finite von Neumann algebras. J. Operator Theory 9 (1983), 253-268.

[P86] Popa, S., Correspondences. INCREST preprint No. 56/1986, www.math.ucla.edu/ $\sim$ popa/preprints.html.

[P90] Popa, S., Free independent sequences in type $\mathrm{II}_{1}$ factors and related problems. Astérisque 232 (1995), 187-202.

[P91] Popa, S., Markov traces on universal Jones algebras and subfactors of finite index. Invent. Math. 111 (1993), 375-405.

[P94] Popa, S., Classification of subfactors of type II. Acta Math. 172 (1994), 163-255.

[P97] Popa, S., Some properties of the symmetric enveloping algebras with applications to amenability and property T. Doc. Math. 4 (1999), 665-744.

[P01a] Popa, S., Some rigidity results for non-commutative Bernoulli shifts. J. Funct. Anal. 230 (2006), 273-328.

[P01b] Popa, S., On a class of type $\mathrm{II}_{1}$ factors with Betti numbers invariants. Ann. of Math. 163 (2006), 809-889.

[P03] Popa, S., Strong Rigidity of $\mathrm{II}_{1}$ Factors Arising from Malleable Actions of $w$-Rigid Groups I. Invent. Math. 165 (2006), 369-408.

[P04a] Popa, S., Strong Rigidity of $\mathrm{II}_{1}$ Factors Arising from Malleable Actions of $w$-Rigid Groups II. Invent. Math. 165 (2006), 409-451.

[P04b] Popa, S., Some computations of 1-cohomology groups and construction of non orbit equivalent actions. J. Inst. Math. Jussieu 5 (2006), 309-332.

[P04c] Popa, S., Deformation, Rigidity and Classification Results for Group Actions and von Neumann Algebras . Course at College de France, Nov. 2004.

[P05] Popa, S., Cocycle and orbit equivalence superrigidity for malleable actions of w-rigid groups. math.GR/0512646.

[P06] Popa, S., On the superrigidity of malleable actions with spectral gap. In preparation.

[PSa03] Popa, S., Sasyk, R., On the cohomology of Bernoulli actions. Ergodic Theory Dynam. Systems, to appear; math.OA/0310211.

[PSS04] Popa, S., Sinclair, A., Smith, R., Perturbation of subalgebras of type $\mathrm{II}_{1}$ factors. $J$. Funct. Anal. 213 (2004), 346-379.

[PV06] Popa, S., Vaes, S., Strong rigidity of generalized Bernoulli actions and computations of their symmetry groups. Preprint; math.OA/0605456.

[R94] Radulescu, F., Random matrices, amalgamated free products and subfactors of the von Neumann algebra of a free group. Invent. Math. 115 (1994), 347-389.

[Sc81] Schmidt, K., Amenabilty, Kazhdan's property T, strong ergodicity and invariant means for ergodic group-actions. Ergodic Theory Dynam. Systems 1 (1981), 223-236.

[Sch63] Schwartz, J., Two finite, non-hyperfinite, non-isomorphic factors. Comm. Pure Appl. Math. 16 (1963), 19-26.

[S00] Shalom, Y., Rigidity of commensurators and irreducible lattices. Invent. Math. 141 (2000), 1-54.

[S05] Shalom, Y., Measurable group theory. In Proceedings of the European Congress of Mathematics (Stockholm, 2004), EMS Publishing House, Zürich 2004, 391-423. 
[Sh04] Shlyakhtenko, D., On the classification of Full Factors of Type III. Trans. Amer. Math. Soc. 356 (2004) 4143-4159.

[Si55] Singer, I. M., Automorphisms of finite factors. Amer. J. Math. 177 (1955), 117-133.

[T03] Takesaki, M., Theory of operator algebras. Encyclopaedia Math. Sci. 127, SpringerVerlag, Berlin 2003.

[Th06] Thomas, S., Popa Superrigidity and Countable Borel Equivalence Relations. In preparation.

[To06] Tornquist, A., Orbit equivalence and actions of $\mathbb{F}_{n}$. J. Symbolic Logic 71 (2006), 265-282.

[V06] Vaes, S., Rigidity results for Bernoulli actions and their von Neumann algebras (after Sorin Popa). Séminaire Bourbaki, exposé 961; Astérisque, to appear.

[Va05] Valette, A., Group pairs with relative property (T) from arithmetic lattices. Geom. Dedicata 112 (2005), 183-196.

[Ve71] Vershik, A., Nonmeasurable decompositions, orbit theory, algebras of operators. Dokl. Akad. Nauk SSSR 199 (1971), 1218-1222.

[Vo90] Voiculescu, D., Circular and semicircular systems and free product factors. In Operator algebras, unitary representations, enveloping algebras, and invariant theory (Paris, 1989), Prog. Math. 92, Birkhäuser, Boston, 1990, 45-60.

[Vo94] Voiculescu, D., The analogues of entropy and of Fisher's information theory in free probability II. Invent. Math. 118 (1994), 411-440 .

[Z80] Zimmer, R., Strong rigidity for ergodic actions of semisimple Lie groups. Ann. of Math. 112 (1980), 511-529.

[Z84] Zimmer, R., Ergodic Theory and Semisimple Groups. Monogr. Math. 81, Birkhäuser, Basel 1984.

[Z91] Zimmer, R., Superrigidity, Ratner's theorem and the fundamental group. Israel J. Math. 74 (1991), 199-207.

Department of Mathematics, University of California, Los Angeles, CA 90095-155505,

U.S.A.

E-mail: popa@math.ucla.edu 